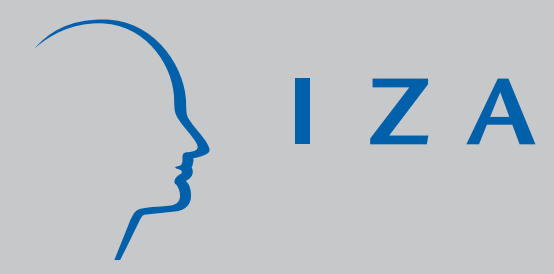

IZADP No. 2056

Simultaneous Search with Heterogeneous Firms and Ex Post Competition

Pieter A. Gautier

Ronald P. Wolthoff

March 2006 


\title{
Simultaneous Search with Heterogeneous Firms and Ex Post Competition
}

\author{
Pieter A. Gautier \\ Free University of Amsterdam, \\ Tinbergen Institute and IZA Bonn \\ Ronald P. Wolthoff \\ Free University of Amsterdam \\ and Tinbergen Institute
}

\section{Discussion Paper No. 2056 \\ March 2006}

\author{
IZA \\ P.O. Box 7240 \\ 53072 Bonn \\ Germany
}

\author{
Phone: +49-228-3894-0 \\ Fax: +49-228-3894-180 \\ Email: iza@iza.org
}

\begin{abstract}
Any opinions expressed here are those of the author(s) and not those of the institute. Research disseminated by IZA may include views on policy, but the institute itself takes no institutional policy positions.

The Institute for the Study of Labor (IZA) in Bonn is a local and virtual international research center and a place of communication between science, politics and business. IZA is an independent nonprofit company supported by Deutsche Post World Net. The center is associated with the University of Bonn and offers a stimulating research environment through its research networks, research support, and visitors and doctoral programs. IZA engages in (i) original and internationally competitive research in all fields of labor economics, (ii) development of policy concepts, and (iii) dissemination of research results and concepts to the interested public.
\end{abstract}

IZA Discussion Papers often represent preliminary work and are circulated to encourage discussion. Citation of such a paper should account for its provisional character. A revised version may be available directly from the author. 
IZA Discussion Paper No. 2056

March 2006

\section{ABSTRACT \\ Simultaneous Search with Heterogeneous Firms and Ex Post Competition*}

We study a search model where workers can send multiple applications to high and low productivity firms. Firms that compete for the same candidate can increase their wage offers as often as they like. We show that there is a unique equilibrium where workers mix between sending both applications to the high and both to the low productivity sector. Efficiency requires however that they apply to both sectors because then the coordination frictions are lowest. For many configurations, the equilibrium outcomes are the same under directed and random search. Allowing for free entry creates a second source of inefficiency.

JEL Classification: D83, E24, J23, J24, J64

Keywords: directed search, efficiency, coordination frictions

Corresponding author:

Pieter A. Gautier

Department of Economics

Vrije Universiteit Amsterdam

De Boelelaan 1105

1081 HV Amsterdam

The Netherlands

Email: pgautier@feweb.vu.nl

\footnotetext{
* We thank Jaap Abbring, Maarten Janssen, Debby Lanser and seminar participants at the Tinbergen
} Institute. 


\section{Introduction}

We study a portfolio problem where unemployed workers must decide in which sector(s) to search. Workers know the productivity in each sector but learn about the wage at a specific firm after applying there. We allow firms that compete for the same candidate to increase their offers as often as they like.

Specifically, we consider a large labor market with identical workers and a high and a low productivity sector. Within a sector, all firms are identical. Workers can send 0,1 or 2 applications at a cost $k>0$ for each application. Each vacancy that receives one or more candidates randomly picks a candidate and offers the job to him. The other applications are rejected. We are interested in symmetric pure strategy equilibria (in terms of the number of applications) and their efficiency properties. Interestingly, it cannot be an equilibrium for workers to send just one application because then firms have no incentives to offer a positive wage. This is basically the Diamond (1971) paradox. Therefore, if $k$ is sufficiently low, workers always send two applications, hoping to get a positive payoff by receiving two offers. But this on its turn implies that workers will never apply to both sectors $(H L)$ because this strategy is strictly dominated by sending both applications to the low productivity sector $(L L)$. The intuition behind this result is that in order for workers to be willing to apply to the low productivity sector, the expected number of applications must be lower there. However, the expected payoffs of receiving an offer from a high and a low productivity firm is the same as receiving offers from two low productivity firms because a high productivity firm that (Bertrand) competes with a low productivity firm for the same candidate will win and pay the productivity level of the worker at the low productivity firm. So, the worker's payoffs conditional on getting two offers are the same for a worker who sends both applications to the low productivity sector $(L L)$ and a worker who plays $H L$, but the probability of receiving two offers is higher for the first worker. We then show that there is a unique mixed strategy equilibrium where workers send both applications with probability $q_{H H}^{*}$ to the high productivity sector and with probability $1-q_{H H}^{*}$ to the low productivity sector where $q_{H H}^{*}$ depends on the relative productivity and the relative supply of vacancies in each of the sectors. As in Albrecht et al. (2006) there are two coordination problems in the matching process: (1) workers do not know where other workers apply to and (2) firms do not know which candidate other firms consider.

By allowing workers to apply to different sectors, the degree of coordination frictions becomes partly endogenous, even for a given number of applications per worker. Workers do however not internalize the effects of their portfolio choice on the aggregate coordination frictions. They just want to maximize the productivity-weighted probability to receive multiple offers. Therefore, the resulting equilibrium is not efficient and unemployment is too high. An important reason for the inefficiency is that a social planner would like some or all workers to apply to both sectors in order to reduce the coordination problems in the matching process. This does not occur however because the expected payoffs of this strategy are too low, since high productivity firms would either pay the monopsony wage or the productivity level of a low productivity firm in case the worker has two offers. Since the expected payoff of playing $H L$ is independent of high productivity output, workers incentives are distorted. Another source of inefficiency is that because of the 
coordination frictions, the matching function is non-monotonic in the number of applications. When there are relatively few vacancies, the second coordination problem is severe and the matching rate is decreasing in the number of applications. The planner internalizes this while individual workers apply too often to the high productivity sector. A similar problem arises at the academic job market where the top universities typically receive too many applicants.

The fact that search is random and not directed is not driving our inefficiency result, since we show that if the number of firms in the market or the difference in productivity between both sectors is not too large, the equilibrium outcomes are the same as in the equilibrium of the directed search version of our model where firms can post a wage ex ante and workers observe all wages. ${ }^{1}$ The reason for this is the same as the one in Albrecht et al. (2006) where posted wages are zero. They consider the case where all workers and firms are identical and they show that the existence of ex post competition makes it still attractive for workers to apply to firms who offer the monopsony wage. Offering a higher wage then the monopsony wage only marginally increases the number of applicants in expectation, because workers mainly care about the probability to get multiple offers, while the expected firm payoffs in case of a match drop linearly.

In section 3 we also allow for free entry of vacancies. We do this by allowing the output of both sectors to be traded in a competitive goods market where consumers have love-for-variety demand for both types of output and both types are imperfect substitutes. ${ }^{2}$ Now, not only the workers' incentives are distorted, but also firms' incentives are distorted. Vacancy supply in each sector can both be too high or too low while typically, the market assigns too few workers to the high productivity sector. Even if we restrict the planner to playing only $H H$ and $L L$, the inefficiency remains.

There are a couple of papers related to what we do. First, Shimer (2005) and Shi (2002) consider a directed search model with two-sided heterogeneity where workers can only apply to one job and ex post competition is irrelevant. They find that the decentralized market outcome is constrained efficient. Our model reduces to Albrecht et al. (2006) when both sectors have the same productivity. Then, workers randomize between all firms and the possibility of ex post Bertrand competition drives down the ex ante posted wages to zero. In Gautier and Moraga-Gonzalez (2004) workers and firms are also identical and workers only learn about the wage after a firm is contacted. There, wages and the number of applications are determined in a simultaneous move game. Chade and Smith (2004) and Galenianos and Kircher (2005) also consider portfolio problems of workers who can apply to multiple jobs. In the latter paper, all jobs have the same productivity but because firms must commit to their posted wages they respond to the worker's desire to diversify. This desire to diversify is driven by the fact that the expected payoff is equal to the maximum wage offer of a worker and not to the average one. Chade and Smith (2004) is not an equilibrium model but it considers a general class of portfolio problems in the absence of ex post competition. Finally, Davis (2001) analyzes a model in which workers and firms can decide to invest in respectively human capital

\footnotetext{
${ }^{1}$ Usually, the equilibrium in directed search models is constraint efficient, e.g. Moen (1999), Montgomery (1991), Peters (1991).

${ }^{2}$ The fixed vacancy supply case can be considered to be a special case with Leontief demand. Further, if output in both sectors are perfect substitutes, only one good will be produced namely the one where the expected value of a vacancy is highest.
} 
and job quality. Because they cannot capture the full increase of the match surplus generated by these investments, both firms and workers tend to underinvest. In equilibrium there is excessive supply of inferior jobs and inferior workers.

The paper is organized as follows. Section 2 describes the basic version of the model in which the number of vacancies is assumed to be exogenously given. This assumption is relaxed in the extended model in section 3. In section 4 we check whether our conclusions are sensitive to the assumptions we make. Section 5 concludes.

\section{Basic Model}

\subsection{Labor Market}

Consider a labor market with $u$ risk neutral workers and $v$ risk neutral firms. All workers are identical, but the firms are divided into two different types. There are $v_{H}$ high-productivity firms and $v_{L}$ low-productivity firms, with $v=v_{H}+v_{L}$. We refer to those firms as highs and lows. Each firm has exactly one vacancy.

Workers can send zero, one, or two applications at $\operatorname{costs} k>0$. Those applications can be directed to a specific type of vacancy, but workers do not observe ex ante the wage that a particular firm offers. If a worker receives multiple job offers, there is Bertrand competition for his services. Basically, workers face a portfolio problem: they have to decide whether they want to send both applications to high type vacancies, both applications to low type vacancies, or one application to a high type and one to a low type vacancy. We will show that if there are not too many firms in the market and if the productivity of the low type firms is not too small, our results carry over to a directed search setting, where workers observe ex ante the wages at each individual firm.

We make three important further assumptions. First, we assume that the labor market is large, i.e. $u \rightarrow \infty$ and $v \rightarrow \infty$, keeping $\theta_{i} \equiv v_{i} / u$ fixed $\forall i \in\{H, L\}$. For the moment, we assume that $\theta_{H}$ and $\theta_{L}$ are exogenously given. We relax this assumption in section 3. Second, we focus on symmetric equilibria, which means that identical agents must have identical strategies. This excludes equilibria that require a lot of coordination amongst workers, something that seems hard to imagine in a large labor market. Third, we assume like Shimer (2005) that the labor market is anonymous: firms must treat identical workers identically and vice versa. So, a worker's strategy may only be conditioned on the type ( $H$ or $L)$ of the firm.

\subsection{Setting of the Game}

The model that is closest related to ours is the one used in Albrecht et al. (2006). There are two differences: (i) we allow for heterogeneity amongst firms and (ii) search is not fully directed. The setting of the game is as follows:

1. Each vacancy posts a wage mechanism.

2. Workers observe all vacancy types (but not the wage mechanism) and send $a \in\{0,1,2\}$ applications. 
3. Each vacancy that receives at least one application, randomly selects a candidate. Applications that are not selected are returned as rejections.

4. A vacancy with a processed application offers the applicant the job. If the applicant receives more than one offer, the firms in question can increase their bids as often as they like.

5. A worker that receives one job offer will accept that offer as long as the offered wage is non-negative. A worker with two offers will accept the one that gives him the highest wage, or will select a job randomly if the offered wages are equal.

If a type $i$ firm matches with a worker, it produces $y_{i}$ units of output. Without loss of generality we assume that $y_{L}<y_{H}=1$. The payoff of a firm that matches with a worker equals $y_{i}-w$, where $w$ denotes the wage that the firm pays. A worker hired at wage $w$ receives a payoff that is equal to that wage. Workers and firms that fail to match receive payoffs of zero.

\subsection{Decentralized Market}

We start the analysis of the decentralized market by showing that no firm posts a positive wage. This is basically the Diamond (1971) paradox.

Lemma 1 In equilibrium all firms post a wage equal to zero

Proof. Note that workers can direct their applications to a specific kind of vacancy, but not to a particular firm. So, posting a higher wage (or more general: a more generous wage mechanism) does not attract more applicants and does not affect the matching probability. This implies that there is no incentive for a firm to offer the worker more than zero. ${ }^{3}$

A direct result of this lemma is that workers never send only one application.

Corollary 1 No equilibrium exists in which there are workers that only send one application.

Proof. Note that if a worker sends one application, there will never be ex post competition for his services. Firms offer a wage equal to zero, so the worker's payoff always equals $-k$. Hence, applying to one job is strictly dominated by not applying at all and therefore never part of an equilibrium strategy.

Whether a worker applies twice or not at all depends on the cost $k$ of sending an application. For example if $k>0.5$, each worker will decide not to apply, because applying twice costs more than the competitive wage $\left(2 k>1=y_{H}\right)$. On the other hand, all workers apply to two jobs if $k$ is sufficiently small, because this gives a strictly positive expected payoff, while not applying results in a payoff of zero. In this paper we restrict

\footnotetext{
${ }^{3}$ Note that this argument implies that posting a wage equal to zero does not only dominate posting a strictly positive wage, but also all other feasible wage mechanisms.
} 
ourselves to the situation in which $k$ is small enough to guarantee that $a=2$ with probability $1 .{ }^{4}$ In this respect our model differs from Shimer (2005) and Shi (2002) where $a=1$.

Three different strategies are possible: a worker can either apply to two high type vacancies, two low type vacancies, or one high type and one low type of vacancy. Denote the respective probabilities by $q_{H H}, q_{L L}$, and $q_{H L}$, where $q_{H H}+q_{L L}+q_{H L}=1$. Using the fact that each worker uses the same strategies, this implies that the total number of applications to firms of type $i$ is equal to $\left(2 q_{i i}+q_{H L}\right) u$. The expected number of applications a specific vacancy receives, is therefore given by

$$
\phi_{i}\left(q_{i i}, q_{H L}, \theta_{i}\right)=\frac{2 q_{i i}+q_{H L}}{\theta_{i}} .
$$

Since our labor market is large, the actual number of applications to a specific vacancy follows a Poisson distribution with mean $\phi_{i} \cdot{ }^{5}$ Next, consider a single individual who applies to a type $i$ firm. The number of competitors for the job at that firm also follows a Poisson distribution with mean $\phi_{i}$, because there is an infinite number of workers. In case of $n$ other applicants, the probability that the individual in question will get the job equals $\frac{1}{n+1}$. Therefore, the probability that an application to a type $i$ firm results in a job offer equals

$$
\begin{aligned}
\psi_{i} & =\sum_{n=0}^{\infty} \frac{1}{n+1} \frac{e^{-\phi_{i}} \phi_{i}^{n}}{n !} \\
& =\frac{1}{\phi_{i}}\left(1-e^{-\phi_{i}}\right) .
\end{aligned}
$$

Note that this expression is not well defined for $\phi_{i}=0$. For convenience we define $\psi_{i}(0)=\lim _{\phi_{i} \rightarrow 0} \psi_{i}\left(\phi_{i}\right)=$ 1 .

Whether a worker's second application results in an offer does not depend on whether the first application was successful or not. A worker who plays $i j$ (i.e. applies to a type $i$ firm and a type $j$ firm) with $i, j \in\{H, L\}$ therefore has a probability $\psi_{i} \psi_{j}$ of getting two job offers and a probability $\psi_{i}\left(1-\psi_{j}\right)+\psi_{j}\left(1-\psi_{i}\right)$ of getting one job offer. The matching probability of such a worker equals one minus the probability that he does not get a job offer and is therefore equal to $1-\left(1-\psi_{i}\right)\left(1-\psi_{j}\right)$ (see Albrecht et al., 2006 for a proof in the case with homogenous firms). This matching probability is obviously strictly increasing in both $\psi_{i}$ and $\psi_{j}$ and depends on the worker's portfolio choice.

If a worker receives two high job offers, Bertrand competition between the two firms results in a wage equal to $y_{H}=1$. In case of two low offers, the firms increase their bids until the worker's wage equals $y_{L}$. A combination of one high and one low offer also implies a wage of $y_{L}$, because at that wage level the low type firm is no longer willing to increase its bid. This is the standard result from Bertrand competition. As shown above, a worker who receives only one job offer gets a wage equal to zero.

Next, we prove that workers never send one application to a high firm and one to a low firm:

Lemma 2 Workers never play HL, since this strategy is strictly dominated.

\footnotetext{
${ }^{4}$ An explicit expression for the upperbound $K$ on $k$ in that case is derived below.

${ }^{5}$ For ease of exposition we omit the arguments of functions whenever this does not lead to confusion.
} 
Proof. The expected payoff for a worker who plays $H L$ is $\psi_{H} \psi_{L} y_{L}-2 k$, i.e. the probability that he receives two job offers times the productivity of the low type firm minus the application cost. Likewise, the expected payoffs of playing $H H$ and $L L$ are $\psi_{H}^{2} y_{H}-2 k$ and $\psi_{L}^{2} y_{L}-2 k$ respectively. Suppose that $\psi_{H} \geq \psi_{L}$. In that case all workers play $H H$, since that strategy gives a strictly higher payoff than $H L$ and $L L$. This however implies that $\phi_{L}=0$ and thus that $\psi_{L}=1$, which contradicts $\psi_{H} \geq \psi_{L}$. Hence, in equilibrium it must be the case that $\psi_{L}>\psi_{H}$. Then, playing $L L$ gives a strictly higher payoff than $H L$. So, $H L$ is strictly dominated.

Lemma 2 implies that there are only two potential pure strategy equilibria, one in which workers send both applications to high type firms and one in which they send both applications to low type firms. In the following Proposition we show that the latter can never be an equilibrium, while the former can, but only under certain conditions.

Proposition 1 A pure strategy equilibrium for the workers only exists if

$$
\frac{\theta_{H}^{2}}{4}\left(1-\exp \left(-\frac{2}{\theta_{H}}\right)\right)^{2}>y_{L}
$$

In that case $q_{H H}^{*}=1$.

Proof. There are two possibilities for a pure strategy (in terms of the sector to apply to): (i) $q_{L L}=1$ and (ii) $q_{H H}=1$. The case in which $q_{H L}=1$ is ruled out by lemma 2 . Since we only consider strategies in which workers apply twice, we can safely ignore the application cost $k$ in this proof. This parameter only plays a role in comparing the payoffs of strategies that differ in the number of applications sent.

(i) Suppose that $q_{L L}=1$. The expected payoff for the workers then is $\psi_{L}^{2} y_{L}<y_{L}$. A worker who deviates and applies twice to a high firm gets two high job offers and therefore a wage that equals $y_{H}=1>y_{L}$. So, a profitable deviation exists, which implies that $q_{L L}=1$ is not an equilibrium.

(ii) Suppose that $q_{H H}=1$. The expected payoff for the workers is in that case $\psi_{H}^{2}=\frac{\theta_{H}^{2}}{4}\left(1-\exp \left(-\frac{2}{\theta_{H}}\right)\right)^{2}$. Deviating to $L L$ gives a wage $y_{L}$ for sure. So $q_{H H}^{*}=1$ is an equilibrium if condition (3) holds.

Hence, we have a pure strategy equilibrium in which all firms post a wage equal to zero and all workers apply twice to high type vacancies if condition (3) holds. This condition imposes very low upperbounds on $y_{L}$ for any reasonable value of $\theta_{H}$ (e.g. $\theta_{H}=0.5$ implies $y_{L}<0.06$ ). The case in which the condition does not hold is therefore more interesting. Then, we only have a mixed strategy equilibrium.

Proposition $2 A$ unique mixed strategy equilibrium exists for any $\theta_{H}>0, \theta_{L}>0$, and $y_{L} \in(0,1)$ such that $\frac{\theta_{H}^{2}}{4}\left(1-\exp \left(-\frac{2}{\theta_{H}}\right)\right)^{2}<y_{L}$. This equilibrium can be characterized by the value $q_{H H}^{*}$ that solves the equality $\psi_{H}^{2}=\psi_{L}^{2} y_{L}$.

Proof. Again, we can rule out the possibility that workers play $H L$ because of lemma 2 . The only mixed strategy equilibrium that can exist is therefore one in which the workers are indifferent between playing $H H$ and $L L$, i.e. $\psi_{H}^{2}=\psi_{L}^{2} y_{L}$. 
If we substitute $q_{L L}=1-q_{H H}$, the only free parameter in this condition is $q_{H H}$. To see that a unique equilibrium value $q_{H H}^{*}$ exists, note that the left hand side of the condition is continuous and strictly decreasing in $q_{H H}$, while the right hand side is continuous and strictly increasing in $q_{H H}$ (see Figure 1). Furthermore, we have

$$
\lim _{q_{H H} \rightarrow 0} \psi_{H}^{2}=1>\frac{\theta_{L}^{2}}{4}\left(1-\exp \left(-\frac{2}{\theta_{L}}\right)\right)^{2} y_{L}=\lim _{q_{H H} \rightarrow 0} \psi_{L}^{2} y_{L}
$$

and

$$
\lim _{q_{H} \rightarrow 1} \psi_{H}^{2}=\frac{\theta_{H}^{2}}{4}\left(1-\exp \left(-\frac{2}{\theta_{H}}\right)\right)^{2}<y_{L}=\lim _{q_{H H} \rightarrow 1} \psi_{L}^{2} y_{L} .
$$

Applying the Intermediate Value Theorem now shows that there exists a unique value $0<q_{H H}^{*}<1$ such that $\psi_{H}^{2}=\psi_{L}^{2} y_{L}$ holds.

Unfortunately, we are not able to derive an explicit expression for $q_{H H}^{*}$. Figure 1 shows the equilibrium as the intersection point of the $\psi_{H}^{2}$-curve and the $\psi_{L}^{2} y_{L}$-curve for $\theta_{H}=\theta_{L}=0.5$ and $y_{L}=0.5$. For those values $63 \%$ of the workers plays $H H$, while $37 \%$ plays $L L$.

In equilibrium the expected payoff for a worker equals $\psi_{H}^{2}-2 k=\psi_{L}^{2} y_{L}-2 k$. The requirement that this value should be larger than the payoff of not applying at all, i.e. zero, implies that $k$ should be smaller than $\frac{1}{2} \psi_{H}^{2}=\frac{1}{2} \psi_{L}^{2} y_{L}$. This assumption seems reasonable. It is hard to imagine that the cost of a particular application exceeds half the expected wage of a job.

The equilibrium depends on three exogenous parameters, $\theta_{H}, \theta_{L}$, and $y_{L}$. The effect of a change in one of these parameters on the equilibrium values of $q_{H H}^{*}, \phi_{i}^{*}$ and $\psi_{i}^{*}$ is summarized by the following Proposition.

Proposition 3 (i) $q_{H H}^{*}, \psi_{H}^{*}$, and $\psi_{L}^{*}$ are strictly increasing in $\theta_{H}$, while $\phi_{H}^{*}$ and $\phi_{L}^{*}$ are strictly decreasing in $\theta_{H}$. (ii) $\psi_{H}^{*}$ and $\psi_{L}^{*}$ are strictly increasing in $\theta_{L}$, while $q_{H H}^{*}, \phi_{H}^{*}$ and $\phi_{L}^{*}$ are strictly decreasing in $\theta_{L}$. (iii) $\psi_{H}^{*}$ and $\phi_{L}^{*}$ are strictly increasing in $y_{L}$, while $q_{H H}^{*}, \psi_{L}^{*}$ and $\phi_{H}^{*}$ are strictly decreasing in $y_{L}$.

Proof. See appendix.

This result is intuitive. A ceteris paribus increase in the number of high productivity firms increases the probability that an application to a firm of this type results in a match. Therefore, it becomes more attractive to play $H H$, resulting in a higher value of $q_{H}^{*}$. The effect of the increase in the number of firms however dominates this increase in $q_{H H}^{*}$, such that the probability to get a job offer increases. Since less workers apply to low productivity firms, the probability to get a job offer increases there as well.

The effect of an increase in the number of low firms is similar: more workers apply to this type of vacancies and the probability to get a job offer increases at both the high and the low types of firms. A change in the productivity of the low firms does not directly affect the probability to match, but it does affect the payoff in case a worker receives two job offers from low type firms. A higher productivity of the low productivity firms is therefore associated with more applications to these firms (see also Figure 2). The number of low firms does however not change, which implies that the probability to get a job offer decreases. 


\subsection{Efficiency}

In the mixed strategy equilibrium that we derived in the previous subsection, a fraction $q_{H}^{*}$ of the workers matches with probability $1-\left(1-\psi_{H}^{*}\right)^{2}$ to a high firm and produce output $y_{H}=1$. The remaining workers match with probability $1-\left(1-\psi_{L}^{*}\right)^{2}$ to a low firm and produce output $y_{L}$. The total output $Y^{*}$ per worker in this equilibrium is therefore given by

$$
Y^{*}=q_{H H}^{*}\left(1-\left(1-\psi_{H}^{*}\right)^{2}\right)+\left(1-q_{H H}^{*}\right)\left(1-\left(1-\psi_{L}^{*}\right)^{2}\right) y_{L} .
$$

The main question of this paper is whether the equilibrium value $q_{H H}^{*}$ is constrained efficient. In order to answer this question we consider a social planner who maximizes total output in the economy. The planner cannot eliminate the coordination frictions, but he can decide to which firms the workers apply. In other words, he can control $q_{H H}, q_{L L}$, and $q_{H L}$. In section 3 we allow for free entry of vacancies and let the planner also determine $\theta_{H}$ and $\theta_{L}$.

Note that although in the decentralized market nobody would ever play $H L$, the social planner can have an incentive to let people play this strategy. Workers do not play $H L$ themselves, because they are only interested in getting two job offers in the same sector. However, from the planner's point of view two job offers to the same worker is always inefficient, because in that case one firm remains unmatched, while it could have matched with a worker without any job offers. Hence, all workers ideally receive only one job offer. The planner can however not coordinate the job offers, so the only way in which he can reduce the coordination problem is by spreading the applications as much as possible, i.e. by playing $H L$.

We assume that the social planner can also decide which job a worker will take if he receives both a high and a low job offer. Suppose that he sends a fraction $\alpha$ of those workers to the high type firm and a fraction $1-\alpha$ to the low type firm. Then we can derive $\chi_{i j}^{k}, i, j, k \in\{H, L\}$, which represents the probability that playing $i j$ results in a match with a type $k$ firm. These probabilities are functions of $\alpha, \psi_{H}$, and $\psi_{L}$ :

$$
\begin{aligned}
\chi_{H H}^{H} & =1-\left(1-\psi_{H}\right)^{2} \\
\chi_{H L}^{H} & =\alpha \psi_{H} \psi_{L}+\psi_{H}\left(1-\psi_{L}\right) \\
\chi_{L L}^{L} & =1-\left(1-\psi_{L}\right)^{2} \\
\chi_{H L}^{L} & =(1-\alpha) \psi_{H} \psi_{L}+\psi_{L}\left(1-\psi_{H}\right) .
\end{aligned}
$$

The remaining probabilities, like $\chi_{H H}^{L}$, are equal to zero. Using this notation, we can write the per-worker output created by the high and the low types firms as

$$
Y_{H}=q_{H H} \chi_{H H}^{H}+q_{H L} \chi_{H L}^{H}
$$

and

$$
Y_{L}=\left(q_{L L} \chi_{L L}^{L}+q_{H L} \chi_{H L}^{L}\right) y_{L} .
$$

This implies that the social planner wants to solve the following maximization problem:

$$
\max _{q_{H H}, q_{L L}, q_{H L}, \alpha} q_{H H} \chi_{H H}^{H}+q_{H L} \chi_{H L}^{H}+\left(q_{L L} \chi_{L L}^{L}+q_{H L} \chi_{H L}^{L}\right) y_{L}
$$


subject to $q_{H H}+q_{L L}+q_{H L}=1$.

Solving this maximization problem would give us the optimal values $q_{i j}^{* *}$ and $\alpha^{*}$, which can be used to calculate $Y^{* *}$, the level of output in that case. However, the noninvertibility of $\psi_{i}$, and thus of $\chi_{i j}^{i}$, prevents us from finding an explicit solution for these parameters. We therefore maximize equation (10) numerically. ${ }^{6}$

The most important difference between the decentralized market and the social planner concerns workers playing $H L$. In the decentralized market nobody plays $H L$, while the social planner imposes this strategy on a large group of workers. For many values of $\left\{\theta_{H}, \theta_{L}, y_{L}\right\}$ the planner even lets all workers play this strategy. This is for example the case for $\theta_{H}=\theta_{L} \leq 0.5$ and $y_{L} \in(0,1)$. The planner only considers $H H$ and $L L$ if (i) the productivity of the $L$-types firms is very low, (ii) the number of firms in the market is very large, or (iii) there is a large difference between the number of high type firms and the number of low type firms. We find that $\alpha$ should be equal to 1 in order to maximize the total output, irrespective of the values of $\theta_{H}$ and $\theta_{L}$. So, if a worker receives a job offer from both the high and the low firm, he must always take the job at the high type firm because his marginal productivity is higher there.

Next, we consider the ratio $\frac{Y^{*}}{Y^{* *}}$, i.e. the ratio between the total output in the decentralized equilibrium and the output level created by the social planner. This ratio is displayed in Figure 3. The first thing that strikes is that the decentralized equilibrium is in general not efficient. The output in the decentralized market is only equal to the optimal level for very small values of $y_{L}$ or for $y_{L}=1$. The former case is exactly the situation in which there is a pure strategy equilibrium with all workers applying to high type firms. In the latter case, there is essentially no difference between high and low firms. This suggests that the introduction of heterogeneity distorts incentives.

The model we discuss in this section has two important characteristics that could both potentially cause the inefficiency: (i) the fact that workers in the decentralized market never play $H L$, while the social planner does and (ii) the fact that workers can not direct their applications to specific firms. It is important to note that these two characteristics are not solely responsible for the inefficiency. For example, if we constrain the social planner by not allowing him to let workers play $H L$, then still the decentralized market outcome is not fully efficient, although the level of inefficiency becomes negligible. Also the second characteristic is not fully responsible for the inefficiency. General expressions for an equilibrium in a directed search framework are hard to derive, but the equilibrium outcomes of our model coincide with the equilibrium outcomes of a directed search model for many values of $\theta_{H}, \theta_{L}$, and $y_{L}$, as we state in the following proposition.

Proposition 4 Assume that $k$ small enough to guarantee that all workers send two applications. ${ }^{7}$ Then, for $\theta_{H}$ and $\theta_{L}$ sufficiently small or for $y_{L}$ sufficiently large, the equilibrium outcomes described in section 2.3 are the same as in the directed search version of our model where workers observe all wages before they apply.

\footnotetext{
${ }^{6}$ The numerical results in this paper are obtained using Ox version 3.40 (see Doornik, 2002).

${ }^{7}$ Under directed search we can have an equilibrium with $a=1$ for some values of $k$. Since this is a special case of the model described in Shimer (2005), we focus on sufficiently low values of $k$ such that $a=2$.
} 
Proof. See appendix.

Figure 4 shows for which values of $\theta_{H}=\theta_{L}=\frac{1}{2} \theta$ and $y_{L}$ the random search equilibrium values are the same as the directed search equilibrium values. As we prove in the appendix, only a low type firm can have an incentive to deviate from posting a wage equal to zero. It posts a positive wage if it cannot attract enough applications otherwise. This is the case if there are many other firms in the market or if the low type firms have a low productivity, which makes it unattractive for the workers to apply there. So, under directed search with multiple applications and firm heterogeneity, the standard positive relation between posted wages and productivity breaks down. In Postel-Vinay and Robin (2002) this happens for similar reasons. In their model, workers agree to accept a lower initial wage at high productive firms because of future possibilities of wage increases through Bertrand competition with rival firms. In the directed search version of our model, high productivity firms always get away with posting the reservation wage while low productivity firms do not because the payoff of receiving multiple offers from high productivity firms is more attractive than from low productivity firms.

The fact that the equilibrium values under random search and directed search can coincide implies that the inefficiency of the decentralized equilibrium can not be eliminated by making search fully directed.

The social planner does not only generate a higher level of social welfare but also a lower unemployment rate than the market, as is shown in Figure 5 for $\theta_{H}=\theta_{L}=0.5$. In the planner's solution approximately one third of the workers remains unemployed. This unemployment rate does not depend on $y_{L}$, reflecting the fact that the social planner always plays $H L$ and $\alpha=1$ for the chosen values of $\theta_{H}$ and $\theta_{L}$. On the other hand, the unemployment rate in the market does depend on $y_{L}$ : it decreases from 0.57 for $y_{L}=0$ to 0.32 for $y_{L}=1$ and is always higher than in the social planner's solution. The intuition behind this result is simple: for small values of $y_{L}$ (almost) all workers in the market play $H H$, which causes large coordination frictions and thus a high unemployment rate. If $y_{L}$ increases, a larger fraction of the workers starts to apply to low type vacancies (see Figure 2). This reduces the coordination frictions, since the same number of applications is now spread over more vacancies. As a result, the number of workers who fail to match decreases. The social planner minimizes the coordination frictions by letting everybody play $H L$.

Figure 6 shows the ratio between the number of matches in the high and the low sector. Again, this ratio is constant for the social planner. In the market this ratio is very high for low values of $y_{L}$, which is caused by the fact that (almost) all workers play $H H$ in that case. The low value of $y_{L}$ implies that a worker can hardly earn anything in the low sector, even if he gets two offers. Therefore, all workers try to get two offers in the high sector, even though the probability that this occurs is rather small. If $y_{L}$ increases, the ratio between the number of matches in the high and the low sector decreases, eventually becoming equal to one for the homogenous case, i.e. $y_{L}=1$.

To sum up, for a fixed supply of vacancies the market equilibrium is inefficient mainly because workers never play $H L$. Therefore, the coordination frictions are larger than necessary. Galenianos and Kircher 
(2005) derive a similar result under a different wage mechanism (no ex post competition, directed search and full commitment). Finally, note that in Albrecht et al. (2006) this inefficiency is absent but they show that entry is excessive when workers apply to multiple jobs. In this section we fixed $\theta_{i}$, so their inefficiency does not arise here. In the next section we relax this assumption.

\section{The Goods Market and Free Entry}

\subsection{Setting of the Game}

The aim of this section is to investigate whether heterogeneity distorts entry decisions under multiple applications. Therefore, we extend the basic model by introducing a competitive goods market and free entry of firms. Both types of firms now produce the same amount of output in case of a match $\left(y_{H}=y_{L}=1\right)$, but the value of these outputs on the goods market may differ. Those values are denoted by $p_{H}=1$ (after normalization) and $p_{L}$ respectively. ${ }^{8}$ The demand on the goods market is determined by the workers who receive utility from consuming the high and the low commodity according to the following Cobb-Douglas utility function with the exogenously given constant $0.5<\lambda<1:^{9}$

$$
u\left(x_{H}, x_{L}\right)=x_{H}^{\lambda} x_{L}^{1-\lambda}
$$

where $x_{i}$ represents the consumption of commodity $i$. Consumers maximize this utility function under the budget constraint

$$
x_{H}+p_{L} x_{L} \leq w,
$$

where $w$ denotes the wage of the worker. Basically, output from both sectors is traded in a competitive goods market where $\lambda$ reflects the relative preference for the $H$-good. Here both goods are imperfect substitutes and therefore strictly positive quantities of both goods are consumed.

Before creating a job opening, firms need to buy one unit of installment capital which $\operatorname{costs} c_{H}$ for high type firms and $c_{L}$ for low type firms. If a firm matches with a worker, then it can use the value of the output to cover these costs. Otherwise, it incurs a loss. We assume free entry of vacancies. Hence, risk-neutral firms enter until the point where expected benefits are zero. The other characteristics of the model remain the same. Workers still send two applications and firms can increase their initial bid in case their candidate receives multiple offers.

\subsection{Decentralized Market}

Several of the results derived for the basic model carry over to this more extended version. For example, it remains optimal for all firms to initially post a wage equal to zero. Again, if a worker receives two job

\footnotetext{
${ }^{8}$ The assumption $y_{L}=1$ is without loss of generality, since only the total value of the output, i.e. $y_{L} p_{L}$, is relevant in our analysis. Fixing $y_{L}$ to a value different from 1 therefore only implies a rescaling of $p_{L}$.

${ }^{9}$ Note that the labels high and low no longer refer to the productivity of a firm, since the productivity is assumed to be the same for both types. We nevertheless stick to these labels in order to keep notation consistent. Instead, one can interpret the labels in the following way: high type firms create a commodity that has a heavier weight $\left(\lambda>\frac{1}{2}\right)$ in $(11)$ than the commodity created by the low type firms $\left(1-\lambda<\frac{1}{2}\right)$.
} 
offers, the firms will increase their bids and Bertrand competition pushes the wages to the marginal product. Therefore, the expected wage of a worker who applies twice to a type $i$ firm is equal to $\psi_{i}^{2} p_{i}$, the probability of receiving two job offers multiplied by the value of the output of a type $i$ firm.

The main difference with the model of the previous section is that workers playing $H L$ and receiving two job offers can now be hired by either the high or the low type firm. Which firm hires depends on the value of $p_{L}$, which now is an endogenous variable. As long as $p_{L}<1$, the high type firm wins the Bertrand game and hires the workers at a wage $p_{L}$. On the other hand, if $p_{L}>1$ the worker matches with the low type firm at a wage equal to 1 . In the case that $p_{L}=1$ both firms employ the worker with probability $\frac{1}{2}$. Hence, the expected wage of a worker who plays $H L$ is $\psi_{H} \psi_{L} \min \left\{1, p_{L}\right\}$.

However, again one can show that $H L$ is dominated by either $H H$ or $L L$. The proof is similar to the one in Lemma 2. Only if $p_{L}=1$ and $\psi_{H}=\psi_{L}$, workers are indifferent between playing $H H, L L$, and $H L$, but this is only because in that case all jobs are identical. In all other cases, workers will only consider playing $H H$ and $L L$.

A firm of type $i$ has a positive revenue if it attracts at least one applicant and if the worker to which it offers the job, does not receive a second job offer. The first event happens with probability $\left(1-e^{-\phi_{i}}\right)$, while the probability of the latter equals $\left(1-\psi_{i}\right) .{ }^{10}$ Therefore, the expected profit of such a firm equals

$$
\pi_{i}=\left(1-e^{-\phi_{i}}\right)\left(1-\psi_{i}\right) p_{i}-c_{i},
$$

which under free entry is equal to zero in equilibrium. From this, one can see that an equilibrium in which $H L$ is not strictly dominated, i.e. with $p_{L}=1$ and $\psi_{H}=\psi_{L}$, can only arise if $c_{H}=c_{L}$.

In equilibrium, the ratio of the prices of the commodities must equal the (absolute value of the) marginal rate of substitution (MRS):

$$
\frac{p_{L}}{p_{H}}=\left.\frac{\partial U / \partial x_{L}}{\partial U / \partial x_{H}}\right|_{x_{H}=Y_{H}, x_{L}=Y_{L}}=\frac{1-\lambda}{\lambda} \frac{Y_{H}}{Y_{L}} .
$$

The expected per-worker output created by the high type firms is $q_{H H}\left(1-\left(1-\psi_{H}\right)^{2}\right)$, while the low type firms produce $\left(1-q_{H H}\right)\left(1-\left(1-\psi_{L}\right)^{2}\right)$ per worker. So, equation (13) is equivalent to

$$
p_{L}=\frac{1-\lambda}{\lambda} \frac{q_{H H}}{1-q_{H H}} \frac{1-\left(1-\psi_{H}\right)^{2}}{1-\left(1-\psi_{L}\right)^{2}}
$$

Summarizing we can define the equilibrium as follows:

Definition 1 An equilibrium in the decentralized market is a tuple $\left\{p_{L}, \theta_{H}, \theta_{L}, q_{H}\right\}$ such that the following four conditions hold:

$$
\begin{gathered}
\psi_{H}^{2}=\psi_{L}^{2} p_{L} \\
p_{L}=\frac{1-\lambda}{\lambda} \frac{q_{H H}}{1-q_{H H}} \frac{1-\left(1-\psi_{H}\right)^{2}}{1-\left(1-\psi_{L}\right)^{2}}
\end{gathered}
$$

\footnotetext{
${ }^{10}$ Due to the infinite size of the labor market, these events are independent.
} 


$$
\begin{aligned}
& \left(1-e^{-\phi_{H}}\right)\left(1-\psi_{H}\right)=c_{H} \\
& \left(1-e^{-\phi_{L}}\right)\left(1-\psi_{L}\right)=\frac{c_{L}}{p_{L}}
\end{aligned}
$$

Equation (14) represents the indifference condition for the workers, while equation (15) makes sure that the price of the low commodity equals the MRS. Equation (16) and (17) are the zero-profit conditions for the high and low type firms respectively. Next, we can show that there is a unique equilibrium.

Proposition 5 In a decentralized market a unique equilibrium $\left\{p_{L}^{*}, \theta_{H}^{*}, \theta_{L}^{*}, q_{H}^{*}\right\}$ exists $\forall 0<c_{H}, c_{L}<1$.

Proof. See appendix.

This extended version of the model also has three exogenous parameters, $c_{H}, c_{L}$, and $\lambda$. The following proposition summarizes how the equilibrium is affected by a change in $c_{L}$, the entry cost of the low type firms.

Proposition $6 \phi_{L}^{*}, p_{L}^{*}, q_{H H}^{*}$, and $\theta_{H}^{*}$ are strictly increasing in $c_{L}$, while $\theta_{L}^{*}$ is strictly decreasing in $c_{L}$. Finally, $\phi_{H}^{*}$ is not affected by a change in $c_{L}$.

Proof. See appendix.

The intuition behind Proposition 6 is the following. An increase in $c_{L}$ reduces profits for low type firms and therefore fewer $L$-vacancies are opened. This makes it relatively more attractive to apply to the high type firms, implying that $q_{H H}^{*}$ increases. This pushes up the profits for high type firms, which induces more high type vacancies to be opened. This increase in $\theta_{H}^{*}$ exactly offsets the increase in $q_{H H}^{*}$ such that the probability to get a job after applying to a high type firm remains constant.

The above Proposition also allows us to compare the high type vacancies with the low type vacancies. It turns out that the entry cost is decisive for which type of vacancies receives more applications and gets a higher price for the created output:

Corollary 2 In the decentralized equilibrium, the vacancy type with the higher entry cost receives more applications, provides the worker with a smaller probability of getting a job offer, and has a higher price for the associated produced commodity.

Proof. The proof is similar to the proof of the previous Proposition with $\hat{p}_{L}^{*}$ replaced by $p_{H}^{*} \equiv 1, \hat{\phi}_{L}^{*}$ by $\phi_{H}^{*}, \hat{\psi}_{L}^{*}$ by $\psi_{H}^{*}$, and $\hat{c}_{L}$ by $c_{H}$.

The second exogenously given parameter is the entry cost for the high type firms. An increase in this parameter decreases the price of the low commodity, but increases the expected number of applications to both high and low type vacancies. This is summarized in the following proposition: 
Proposition $7 \phi_{H}^{*}$ and $\phi_{L}^{*}$ are strictly increasing in $c_{H}$, while $p_{L}^{*}$ is strictly decreasing in $c_{H}$.

Proof. The free entry condition for the $H$-firms shows that an increase in $c_{H}$ strictly increases $\phi_{H}^{*}$. This means that $\psi_{H}^{*}$ strictly decreases. For the indifference condition to continue to hold, $\psi_{L}\left(\phi_{L}^{*}\left(p_{L}^{*}\right)\right)^{2} p_{L}^{*}$ has to decrease as well. Since $\psi_{L}\left(\phi_{L}^{*}\left(p_{L}\right)\right)^{2} p_{L}$ is strictly increasing in $p_{L}$ (see proof of Proposition 5), a decrease in $\psi_{H}^{*}$ implies a decrease in $p_{L}^{*}$ and therefore an increase in $\phi_{L}^{*}$.

It is not trivial to analytically derive the effect of an increase in $c_{H}$ on $q_{H H}^{*}, \theta_{H}^{*}$ and $\theta_{L}^{*}$. Since we only have three exogenous parameters we can rely on numerical computations. We find that the above three variables are decreasing in $c_{H}$. Figures 7, 8 and 9 respectively show $q_{H H}^{*}, \theta_{H}^{*}$ and $\theta_{L}^{*}$ as a function of $c_{H}$ for several values of $c_{L}$, where $\lambda=0.6$.

The last exogenously given parameter is the preference parameter $\lambda$, i.e. the share of income that the workers spend on consuming the high commodity. Not surprisingly, an increase in $\lambda$ turns out to have a positive effect on the fraction of workers applying to the high vacancies and on the number of high vacancies. However, it causes a decrease in the number of low vacancies. The change in the number of vacancies exactly offsets the change in $q_{H H}^{*}$, so that the expected number of applications per vacancy does not change

Proposition $8 q_{H H}^{*}, \theta_{H}^{*}$ are strictly increasing in $\lambda$, while $\theta_{L}^{*}$ is strictly decreasing in $\lambda$. A change in $\lambda$ does not affect $\phi_{H}^{*}, \phi_{L}^{*}$, and $p_{L}^{*}$.

Proof. $\phi_{H}^{*}$ is determined by equation (16) only and therefore not affected by a change in $\lambda$. This means that the left hand side of equation (14) remains constant. As we showed in Proposition 5, the right hand side of this equation is strictly increasing in $p_{L}$, implying that $p_{L}^{*}, \psi_{L}^{*}$, and $\phi_{L}^{*}$ do not change either. From the fact that $p_{L}$ remains constant and $\lambda$ increases, we can derive that in equation (15) the factor $\frac{q_{H H}}{1-q_{H} H}$ must increase. Since the first derivative of this expression is strictly positive, this means that $q_{H H}$ has to increase. Now it is straightforward to show that $\theta_{H}^{*}$ must increase and $\theta_{L}^{*}$ must decrease in order to keep $\phi_{H}^{*}$ and $\phi_{L}^{*}$ fixed.

\subsection{Efficiency}

Since we allow for free entry, we can now test whether the number and composition of vacancies is constrained efficient. Specifically, we assume that the social planner can again determine $q_{H H}, q_{L L}, q_{H L}$, and $\alpha$, like in the basic model, but now he can also determine the number and composition of firms in the market, $\theta_{H}$ and $\theta_{L}$. Using the same definitions for $\chi_{i j}^{k}$ as in section 2.4, we can write $Y_{i}$, i.e. total output created by type $i$ firms, as follows:

$$
Y_{H}=q_{H H} \chi_{H H}^{H}+q_{H L} \chi_{H L}^{H}
$$

and

$$
Y_{L}=q_{L L} \chi_{L L}^{L}+q_{H L} \chi_{H L}^{L}
$$


Next, denote the net value of the output per worker by $V$ :

$$
V=p_{H} Y_{H}+p_{L} Y_{L}-\theta_{H} c_{H}-\theta_{L} c_{L}
$$

The social planner is not concerned with redistribution issues. He just wants to maximize social welfare, i.e. the utility that can be obtained from $V$. This implies that he maximizes the indirect utility function associated to the Cobb-Douglas utility function specified in equation (11):

$$
\max _{q_{H H}, q_{L L}, q_{H L}, \alpha, \theta_{H}, \theta_{L}}\left(\frac{\lambda V}{p_{H}}\right)^{\lambda}\left(\frac{(1-\lambda) V}{p_{L}}\right)^{1-\lambda}
$$

under the condition that $q_{H H}+q_{L L}+q_{H L}=1$. Again, the price of the low commodity has to be equal to the marginal rate of substitution. Therefore, we can rewrite equation (18) as follows:

$$
\max _{q_{H H}, q_{L L}, q_{H L}, \alpha, \theta_{H}, \theta_{L}}\left(Y_{H}-\lambda \theta_{H} c_{H}-\lambda \theta_{L} c_{L}\right)\left(\frac{Y_{L}}{Y_{H}}\right)^{1-\lambda} .
$$

The corresponding system of first order conditions cannot be solved analytically. Therefore, we use numerical optimization methods to derive the optimal values $q_{H H}^{* *}, q_{L L}^{* *}, q_{H L}^{* *}, \alpha^{* *}, \theta_{H}^{* *}$, and $\theta_{L}^{* *}$. The results indicate that the optimal value for $q_{L L}^{* *}$ equals 0 , i.e. the social planner does not let workers play $L L{ }^{11}$ The optimal values for $q_{H H}^{* *}=1-q_{H L}^{* *}$ are displayed in Figure 10 for several values of $c_{L}$ and $\lambda=0.6 .{ }^{12}$ Each line shows two clear jumps. The first jump occurs where $c_{H}=c_{L}$, which can be explained by the behavior of $\alpha^{* *}$. This value is always equal to zero for $c_{H}<c_{L}$ and equal to one for $c_{H}>c_{L}$, because when a worker receives both a high and a low type offer, the planner wants the worker to fill the position that is more expensive to create. Ceteris paribus, this jump in $\alpha^{* *}$ at $c_{H}=c_{L}$ increases the probability for a high firm to match and decreases the probability for a low firm to match. Since the output the planner wants to create in the high and the low sector does however not change discontinuously, the positive jump in $\alpha^{* *}$ must be neutralized by a negative jump in $q_{H H}^{* *}$.

The second jump has no clear economic meaning. It is the result of the fact that the social welfare function is non-monotonic in its parameters. The value of $c_{H}$ for which this second jump occurs is negatively related to $\lambda$. For large values of $\lambda$ and $c_{L}$ it can happen that this jump occurs before the point where $c_{H}=c_{L}$. In that case, there is only one jump.

Next, we turn to the important question whether there are too many or too few vacancies created in the decentralized market equilibrium. Albrecht et al. (2006) prove that in their model the market always opens more vacancies than the social planner if the number of applications is fixed, but that there can be either too many or too few vacancies if the number of applications is endogenous. In our model we focus on $a=2$, but the composition of these applications over the sectors is endogenous both for the market and the planner. Unlike in Albrecht et al. (2006), the expected number of applications that a workers sends to a specific

\footnotetext{
${ }^{11}$ This conclusion even holds for $c_{L}$ close to 1 and $\lambda$ close to 0.5 .

${ }^{12}$ Fixing $\lambda$ at a different value, e.g. 0.9 , changes the values of $q_{H H}^{* *}, q_{L L}^{* *}, q_{H L}^{* *}, \alpha^{* *}, \theta_{H}^{* *}$, and $\theta_{L}^{* *}$, but none of the qualitative conclusions in this section.
} 
sector can now be a non-integer value, because he can play mixed strategies with respect to the sectors he applies to. Hence, the heterogeneity amongst the firms gives both the market and the social planner more freedom in choosing the optimal number of applications, even if the total number of applications is fixed.

Figures 11 and 12 respectively display the number of $H$-vacancies and the number of $L$-vacancies created by the market and the planner as a function of the entry cost for type $H$ firms. The entry cost for type $L$ firms is fixed at 0.5, while $\lambda$ is still assumed to be 0.6. ${ }^{13}$ The Figures show that like in Albrecht et al. (2006) either too many or too few vacancies (both high and low) are opened in the decentralized market, depending on the values of the exogenous parameters. For low values of $c_{H}$, the market opens too many vacancies compared to the social optimum. When $c_{H}$ approaches 1 , the reverse holds. In that case, the social planner creates more vacancies than the market.

The intuition for the latter result is the following. If $c_{H}$ approaches 1 in the decentralized market, no high firm is willing to enter, because its expected payoff is negative in that case. However, without supply of the high type commodity, workers can never obtain a positive utility and therefore the entire market collapses: there are no firms active in equilibrium. The social planner finds this undesirable and still lets firms enter the market.

In order to check whether the market is constrained efficient, we compare the ratio between the utility obtained in the decentralized equilibrium (i.e. the indirect utility function evaluated at the equilibrium values) and the utility associated with the social planner's solution. This ratio is displayed by the dashed line in Figure 13 for $\lambda=0.6$. It shows that for small $c_{H}$, market utility is about $80 \%$ of what could be achieved. As $c_{H}$ increases, the inefficiency goes up and when $c_{H}$ approaches 1 , the ratio of market and planner's utility goes to zero.

The intuition for this result is the same as above: in the decentralized market no vacancies are created if $c_{H}$ approaches 1 . The social planner however does create vacancies in that case. So, for $c_{H}$ close enough to 1 , the created output is virtually zero in the decentralized market but strictly positive under the social planner. This implies that the relative efficiency of the decentralized market equilibrium goes to 0 .

To see to what extent this inefficiency is caused by the fact that the planner plays $H L$, we also consider a constrained planner who can only play (a mixture of) $H H$ and $L L$. The efficiency of the decentralized equilibrium relative to this constrained planner's optimum is displayed in Figure 13 by the solid line. The line shows that in this case the inefficiency is almost as large as in the case with the unconstrained planner. This is dramatically different from the model in section 2 where most of the market inefficiency was due to the fact that workers do not play $H L$. It suggests that our results are not driven by the fact that we only have two jobs types, which makes the inefficiency due to not playing $H L$ relatively large.

The conclusions drawn in section 2.4 about employment in the high and the low sector do also not fully carry over to the extended version of the model. As can be seen in Figure 14, the unemployment rate in the

\footnotetext{
${ }^{13}$ Different values of $c_{L}$ and $\lambda$ do not affect the main conclusions.
} 
market is now not always higher than under the social planner. For small values of $c_{H}$ the reverse holds, which directly follows from the fact that the market opens more vacancies than the planner. Figure 15 shows that, except for extremely low values of $c_{H}$ (below 0.02), the planner always generates a higher matching rate in the high sector than the market.

To sum up, the market creates too many vacancies if the vacancy creation costs are low, while it creates to few vacancies if the high type vacancy creation costs are high. As a result of this, the expected number of applications that a high type vacancy receives in a decentralized market is larger than socially optimal for high values of $c_{H}$ and smaller than optimal for low values of $c_{H}$. A similar pattern is found for the expected number of applications received by low type firms. If we restrict the planner to only play $H H$ and $L L$, this conclusion still holds.

\section{Robustness}

In this section we discuss to what extent our results are sensitive to the following three simplifying assumptions we made: (i) a worker cannot send more than two applications, (ii) firms cannot offer the job to more than one applicant, and (iii) firms that compete for the same worker engage in Bertrand competition.

\section{More than two applications}

The first simplifying assumption is that a worker cannot send more than two applications. Allowing workers to apply to more than two jobs makes the analysis more difficult, but does not change the nature of the portfolio problem. Still workers are only interested in the productivity-weighted probability to get more than one job offer, while the social planner wants to spread applications in order to reduce the coordination frictions. So, the fact that we restrict the workers to at most two applications is not driving our main result.

\section{Multiple job offers}

The second assumption is that firms can offer the job to one worker only. This can be restrictive even if we assume that the marginal productivity of a second worker is zero. For example, it can be profitable for a firm to increase its matching probability by offering the same job to more applicants. The drawback of this strategy is that the firm then runs the risk that more than one worker accepts the offer. In that case, the firm has to pay a wage to all the workers it hires, while only one of them can be used in producing output.

Deriving the optimal strategy in such a model is not straightforward. First, timing matters. Suppose that a firm sends two job offers. Initially, it offers a wage equal to zero to both applicants. If one of the candidates has also received another offer, the firm must decide whether it will compete for this worker. The strategy of the firm depends on the result of the second job offer it has made. Therefore, one must make assumptions about the exact moment at which the firm learns the result of each job offer.

One way to solve the timing problem is by assuming that if their candidate has multiple offers, the firms participate in a second-price sealed bid auction, rather than Bertrand competition. ${ }^{14}$ In that case all firms

\footnotetext{
${ }^{14}$ See Julien et al. (2000), Kulti (1999) and Shimer (1999).
} 
submit one bid $w_{i}$ and the bids are revealed simultaneously. The winning firm hires the worker and pays a wage equal to the bid of the competing firm (and zero if there was no competing firm). If firms can make only one job offer, it is optimal for them to bid the productivity level, $w_{i}=y_{i}$. Hence, in that case the payoffs are identical to the payoffs described in the previous sections, i.e. under the assumption of Bertrand competition.

If firms can however make more than one job offer, deriving the optimal wage offer remains difficult. First, it is relevant whether the other offer of the firm's candidate is at a firm with multiple candidates or not. If it is not, the other firm will bid more aggressively. Second, there is no pure strategy equilibrium because each candidate equilibrium wage pair is dominated by either offering one of the candidates a zero wage or offering them $\varepsilon$ more. This is essentially the well known Burdett-Judd (1983) argument. An alternative is the shortlisting assumption of Albrecht et al. (2006) where firms pick a first candidate and a second candidate to whom they offer the job (if she is still available) in case they fail to hire their first candidate. At each of the firms they apply to, workers can be in three possible states: first candidate, second candidate or neither. This makes the algebra tedious but the bottom line is that none of the coordination frictions is eliminated. Even if a firm makes $b$ job offers, it is still possible that it remains unmatched, because all the workers accepted offers from other firms. Moreover, workers still only care about receiving two offers while the planner wants to maximize the output-weighted number of matches. Finally, Gautier et al. (2005) and Kircher (2005) consider the case where firms can consider as many applicants as they like. Kircher shows that if firms commit to their posted wage, the directed search equilibrium is efficient. If firms can increase their initial bids, in case their (final) candidate has multiple offers, the remaining equilibrium remains inefficient.

\section{No Bertrand Competition}

The third assumption concerns Bertrand competition for workers with two offers. Alternatives are for example commitment of the firms to their initial bids, as in Galenianos and Kircher (2005) or offer-beating strategies as in Albrecht et al. (2006). Assuming commitment is basically a restriction on the firm's strategy space. Offer-beating strategies expand the firm's strategy space. Basically, the thread of Bertrand competition can reduce ex post competition and typically multiple equilibria arise. Reducing competition for workers implies that a larger part of the surplus goes to the firms and consequently entry increases. We saw that for low entry cost of $H$-firms, vacancy supply in both sectors was already excessive so reducing ex post competition can never generically increase efficiency.

\section{$5 \quad$ Final Remarks}

We presented a simple model where workers could apply to multiple, heterogeneous jobs. Workers do not apply to firms with the highest expected payoffs for an individual application but rather maximize the value of their portfolio. We also extend the model with free entry.

The resulting equilibrium is not efficient for two reasons. Workers want to maximize the productivity- 
weighted probability to get two job offers, while the planner aims to maximize the productivity-weighted number of matches. This conflict of interest results in too little matches and excessive unemployment. We showed that this result is not driven by the fact that search is random in our model. For a large share of parameter values the posted wages are also zero in the directed search version of our model as in Albrecht et al. (2006).

If we allow for free entry there is a second source of inefficiency. For high creation cost in the high productivity sector, the market creates too little vacancies. If entry cost are high, the risk of Bertrand competition makes firms stop entering the market at a point where the marginal social benefits are still positive. On the other hand if entry cost are low, vacancy creation is excessive because the absence of ex ante competition gives firms too much rents. The vacancy creation distortions can in principle be neutralized by an appropriately chosen firm tax or subsidy scheme. The workers' portfolio distortions are more severe. Governments may have instruments to make one of the sectors more attractive but this will only increase the fraction of workers who send both applications to this sector without increasing the fraction of workers that mixes between sectors.

\section{References}

[1] Albrecht J., P.A. Gautier and S. Vroman, 2006, Equilibrium directed search with multiple applications, Review of Economic Studies, forthcoming.

[2] Burdett, K. And K.L. Judd, 1983, Equilibrium Price Dispersion, Econometrica, vol. 51(4), pp. 955-969.

[3] Chade, H. and L. Smith, 2004, Simultaneous search, Working Paper, Department of Economics, W.P. Carey School of Business, Arizona State University.

[4] Davis, S.J., 2001, The quality distribution of jobs and the structure of wages in search equilibrium, NBER working papers 8434, National Bureau of Economic Research.

[5] Diamond, P., 1971, A model of price adjustment, Journal of Economic Theory, vol. 3, pp. 156-168.

[6] Doonnik, J.A., 2002, Object-Oriented Matrix Programming Using Ox, 3rd ed., Timberlake Consultants Press, London.

[7] Galenianos, M. and P. Kircher, 2005, Directed search with multiple job applications, PIER working paper 05-022, Penn Institute for Economic Research.

[8] Gautier, P.A. And J.L. Moraga-Gonzalez, 2004, Strategic wage setting and random search with multiple applications, Tinbergen Institute discussion paper 04-063/1, Tinbergen Institute.

[9] Gautier, P.A., J.L. Moraga-Gonzalez and M. Nuyens, 2005, Decentralized matching without recruitment constraints, mimeo, Tinbergen Institute. 
[10] Julien, B., J. Kennes And I. King, 2000, Bidding for Labor, Review of Economic Dynamics, vol. 3(4), pp. 619-49.

[11] Kircher, P., 2006, Simultaneous Directed Search with Recall, mimeo, University of Bonn.

[12] Kultti, K., 1999, Equivalence of auctions and posted prices, Games and Equilibrium Behavior, vol. 27(1), pp. 106-113.

[13] Moen E., 1997, Competitive search equilibrium, Journal of Political Economy, vol. 105(2), pp. 385-411.

[14] Postel-Vinay, F. and J.M. Robin, 2002, Equilibrium wage dispersion with worker and employer heterogeneity, Econometrica, vol. 70, pp. 2295-2330.

[15] SHI, S., 2002, A directed search model of inequality with heterogeneous skills and skill-biased technology, Review of Economic Studies, vol. 69, pp. 467-491.

[16] Shimer, R., 1999, Job auctions, mimeo, Princeton University.

[17] Shimer, R., 2005, The assignment of workers to jobs in an economy with coordination frictions, Journal of Political Economy, vol. 113(5), pp. 996-1025. 


\section{Appendix}

\section{A Proofs}

\section{A.1 Proof of Proposition 3}

Proof. (i) First, note that

$$
\frac{\partial \psi_{i}}{\partial \phi_{i}}=\frac{1}{\phi_{i}^{2}}\left(\left(1+\phi_{i}\right) e^{-\phi_{i}}-1\right)<0,
$$

since $e^{\phi_{i}}>1+\phi_{i} \forall \phi_{i}>0$. Using this and $\frac{\partial \phi_{i}}{\partial \theta_{i}}<0$, we can derive that $\frac{\partial \psi_{H}^{2}}{\partial \theta_{H}}=2 \psi_{H} \frac{\partial \psi_{H}}{\partial \phi_{H}} \frac{\partial \phi_{H}}{\partial \theta_{H}}>0$, which means that an increase in $\theta_{H}$ shifts the $\psi_{H}^{2}$-curve in Figure 1 upwards. On the other hand, $\frac{\partial \psi_{L}^{2} y_{L}}{\partial \theta_{H}}=0$, so an increase in $\theta_{H}$ does not affect the $\psi_{L}^{2} y_{L}$-curve. Hence, the intersection point of the two curves moves to the northeast, implying an increase in the equilibrium value $q_{H H}^{*}$ and in the expected payoffs. This means that both $\psi_{H}^{*}$ and $\psi_{L}^{*}$ increase and both $\phi_{H}^{*}$ and $\phi_{L}^{*}$ decrease.

(ii) Note that $\frac{\partial \psi_{L}^{2} y_{L}}{\partial \theta_{L}}=2 \psi_{L} y_{L} \frac{\partial \psi_{L}}{\partial \phi_{L}} \frac{\partial \phi_{L}}{\partial \theta_{L}}>0$. Hence, an increase in $\theta_{L}$ shifts $\psi_{L}^{2} y_{L}$-curve upwards, but does not affect the $\psi_{H}^{2}$-curve. Therefore, $q_{H H}^{*}$ decreases, while $\psi_{H}^{*}$ and $\psi_{L}^{*}$ increase and, consequently, $\phi_{H}^{*}$ and $\phi_{L}^{*}$ decrease.

(iii) Finally, an increase in $y_{L}$ shifts the $\psi_{L}^{2} y_{L}$-curve upwards, but does not affect the $\psi_{H}^{2}$-curve. Therefore, $q_{H H}^{*}$ decreases, while $\psi_{H}^{*}$ increases. The latter implies a decrease in $\phi_{H}^{*}$. Since $\theta_{L}$ remains constant, the decrease in $q_{H H}^{*}$ results in an increase in $\phi_{L}^{*}$ and consequently a decrease in $\psi_{L}^{*}$.

\section{A.2 Proof of Proposition 4}

Proof. Suppose that all firms posting a wage equal to zero is not a directed search equilibrium. Then a profitable deviation must exist for either the high type firms or the low types firms. Consider a deviation by a high type firm first. Instead of 0 it posts a strictly positive wage: $w_{H}^{\prime}>0$. Workers now have two additional application strategies: they can send (i) one application to the deviant and the other one to a high firm or (ii) one application to the deviant and the other one to a low firm. Denote the former strategy by $H^{\prime} H$ and the latter by $H^{\prime} L$. The payoff of playing $H^{\prime} H$ equals

$$
\psi_{H}^{\prime} \psi_{H}+\psi_{H}^{\prime}\left(1-\psi_{H}\right) w_{H}^{\prime}
$$

and the payoff of $H^{\prime} L$ equals

$$
\psi_{H}^{\prime} \psi_{L} y_{L}+\psi_{H}^{\prime}\left(1-\psi_{L}\right) w_{H}^{\prime},
$$

where $\psi_{H}^{\prime}$ is defined in the usual way and denotes the probability that an application to the deviant results in a job offer.

Since we consider a large labor market, a specific worker applies with probability zero to the deviant. So, the presence of a deviant does not affect the average number of applications received by the other nondeviant high or low firms. Therefore, the indifference condition $\psi_{H}^{2}=\psi_{L}^{2} y_{L}$ must still hold. By substituting 
$\psi_{H}=\psi_{L} \sqrt{y_{L}}$ in equation (20) and using the fact that $1>\sqrt{y_{L}}>y_{L}$, one can easily see that $H^{\prime} L$ is dominated by $H^{\prime} H$.

In response to the deviation by one of the high firms, workers will adjust their application strategies such that they are indifferent between $H H, L L$ and $H^{\prime} H$. The new equilibrium is therefore defined by the following two equations:

$$
\begin{aligned}
& \psi_{H}^{2}=\psi_{L}^{2} y_{L} \\
& \psi_{H}^{2}=\psi_{H}^{\prime} \psi_{H}+\psi_{H}^{\prime}\left(1-\psi_{H}\right) w_{H}^{\prime}
\end{aligned}
$$

Let $\phi_{H}^{\prime}$ denote the expected number of applications that the deviant receives. Then, by substituting $\psi_{H}^{\prime}=\frac{1}{\phi_{H}^{\prime}}\left(1-e^{-\phi_{H}^{\prime}}\right)$ in the second condition and rearranging the result, we can derive the following relation between the posted wage $w_{H}^{\prime}$ and $\phi_{H}^{\prime}$ :

$$
w_{H}^{\prime}=\frac{1}{1-\psi_{H}}\left(\frac{\phi_{H}^{\prime} \psi_{H}^{2}}{1-e^{-\phi_{H}^{\prime}}}-\psi_{H}\right) .
$$

The first derivative of this function with respect to $\phi_{H}^{\prime}$ equals

$$
\frac{\partial w_{H}^{\prime}}{\partial \phi_{H}^{\prime}}=\frac{\psi_{H}^{2}}{\psi_{H}-1} \frac{e^{-\phi_{H}^{\prime}}+\phi_{H}^{\prime} e^{-\phi_{H}^{\prime}}-1}{e^{-2 \phi_{H}^{\prime}}-2 e^{-\phi_{H}^{\prime}}+1}>0 \forall \phi_{H}^{\prime}>0 .
$$

Hence, $w_{H}^{\prime}$ is a monotonic function of $\phi_{H}^{\prime}$ : the higher the wage set by the deviant, the higher the expected number of applications it receives.

After substituting equation (22), the profit function for a high type deviant equals

$$
\begin{aligned}
\pi_{H}^{\prime} & =\left(1-e^{-\phi_{H}^{\prime}}\right)\left(1-\psi_{H}\right)\left(1-w_{H}^{\prime}\right) \\
& =\left(1-e^{-\phi_{H}^{\prime}}\right)\left(1-\psi_{H}\right)\left(1-\frac{1}{1-\psi_{H}}\left(\frac{\phi_{H}^{\prime} \psi_{H}^{2}}{1-e^{-\phi_{H}^{\prime}}}-\psi_{H}\right)\right) .
\end{aligned}
$$

Deriving this profit function with respect to $\phi_{H}^{\prime}$ yields the following expression:

$$
\frac{\partial \pi_{H}^{\prime}}{\partial \phi_{H}^{\prime}}=e^{-\phi_{H}^{\prime}}-\psi_{H}^{2},
$$

which is a strictly decreasing function of $\phi_{H}^{\prime}$ that equals zero for $\phi_{H}^{\prime}=-2 \log \left(\psi_{H}\right)$. Therefore, the profit function has a global maximum in this point. The corresponding value of $w_{H}^{\prime}$ follows from evaluating equation (22):

$$
w_{H}^{\prime}=\frac{\psi_{H}\left(\psi_{H}^{2}-2 \psi_{H} \log \left(\psi_{H}\right)-1\right)}{\left(1-\psi_{H}\right)^{2}\left(1+\psi_{H}\right)} .
$$

This expression has the same sign as $\psi_{H}^{2}-2 \psi_{H} \log \left(\psi_{H}\right)-1$. The first derivative of this equation is equal to $2\left(\psi_{H}-\log \psi_{H}-1\right)$, which easily can be shown to be positive for all $\psi_{H}$ in the interval $(0,1)$. Together with the fact that $\lim _{\psi_{H} \rightarrow 1} \psi_{H}^{2}-2 \psi_{H} \log \left(\psi_{H}\right)-1=0$, this implies that the right hand side of equation (23) is negative $\forall \psi_{H} \in(0,1)$. Since we do not allow for negative wages, this optimal value of $w_{H}^{\prime}$ is not feasible. Given that the profit is strictly decreasing in $\phi_{H}^{\prime}>-2 \log \left(\psi_{H}\right)$ and that $w_{H}^{\prime}$ is strictly increasing in $\phi_{H}^{\prime}$, the profit function maximization problem therefore has a boundary solution: the deviant maximizes its profit 
by posting $w_{H}^{\prime}=0$. This implies that if all firms post a wage equal to zero, a profitable deviation does not exist for a high type firm.

Now we perform the same analysis for a low type deviant. Suppose that it posts a wage $w_{L}^{\prime}>0$. In that case the payoff of playing $L L^{\prime}$ equals

$$
\psi_{L} \psi_{L}^{\prime} y_{L}+\psi_{L}^{\prime}\left(1-\psi_{L}\right) w_{L}^{\prime}=\psi_{L}^{\prime} w_{L}^{\prime}+\psi_{L}^{\prime} \psi_{L}\left(y_{L}-w_{L}^{\prime}\right)
$$

and the payoff of $H L^{\prime}$ equals

$$
\psi_{H} \psi_{L}^{\prime} y_{L}+\psi_{L}^{\prime}\left(1-\psi_{H}\right) w_{L}^{\prime}=\psi_{L}^{\prime} w_{L}^{\prime}+\psi_{L}^{\prime} \psi_{H}\left(y_{L}-w_{L}^{\prime}\right),
$$

where $\psi_{L}^{\prime}$ denotes the probability that an application to the deviant results in a job offer.

In a similar way as we described above, one can show that the strategy $H L^{\prime}$ is dominated by $L L^{\prime}$. The new equilibrium is therefore defined by the following two indifference conditions:

$$
\begin{aligned}
\psi_{H}^{2} & =\psi_{L}^{2} y_{L} \\
\psi_{L}^{2} y_{L} & =\psi_{L} \psi_{L}^{\prime} y_{L}+\psi_{L}^{\prime}\left(1-\psi_{L}\right) w_{L}^{\prime}
\end{aligned}
$$

Let $\phi_{L}^{\prime}$ denote the expected number of applications that the deviant receives. Then, by substituting $\psi_{L}^{\prime}=\frac{1}{\phi_{L}^{\prime}}\left(1-e^{-\phi_{L}^{\prime}}\right)$ in the second condition and rearranging the result, we can derive the following relation between the posted wage $w_{L}^{\prime}$ and $\phi_{L}^{\prime}$ :

$$
w_{L}^{\prime}=\frac{1}{1-\psi_{L}}\left(\frac{\phi_{L}^{\prime} \psi_{L}^{2} y_{L}}{1-e^{-\phi_{L}^{\prime}}}-\psi_{L} y_{L}\right)
$$

The first derivative of this function with respect to $\phi_{L}^{\prime}$ equals

$$
\frac{\partial w_{L}^{\prime}}{\partial \phi_{L}^{\prime}}=\frac{\left(e^{-\phi_{L}^{\prime}}+\phi_{L}^{\prime} e^{-\phi_{L}^{\prime}}-1\right) \psi_{L}^{2} y_{L}}{\left(e^{-2 \phi_{L}^{\prime}}-2 e^{-\phi_{L}^{\prime}}+1\right)\left(\psi_{L}-1\right)}>0 \forall \phi_{L}^{\prime}>0 .
$$

Hence $w_{L}^{\prime}$ is a monotonic function of $\phi_{L}^{\prime}$ : the higher the wage set by the deviant, the higher the expected number of applications it receives.

The profit function for the deviant equals

$$
\begin{aligned}
\pi_{H}^{\prime} & =\left(1-e^{-\phi_{L}^{\prime}}\right)\left(1-\psi_{L}\right)\left(1-w_{L}^{\prime}\right) \\
& =\left(1-e^{-\phi_{L}^{\prime}}\right)\left(1-\psi_{L}\right)\left(1-\frac{1}{1-\psi_{L}}\left(\frac{\phi_{L}^{\prime} \psi_{L}^{2} y_{L}}{1-e^{-\phi_{L}^{\prime}}}-\psi_{L} y_{L}\right)\right) .
\end{aligned}
$$

Deriving this this profit function with respect to $\phi_{L}^{\prime}$ yields the following expression:

$$
\frac{\partial \pi_{L}^{\prime}}{\partial \phi_{L}^{\prime}}=e^{-\phi_{L}^{\prime}}\left(1-\left(1-y_{L}\right) \psi_{L}\right)-\psi_{L}^{2} y_{L}
$$

which is a strictly decreasing function of $\phi_{L}^{\prime}$ that equals zero for $\phi_{L}^{\prime}=-\log \kappa$, where $\kappa \equiv \frac{\psi_{L}^{2} y_{L}}{1-\left(1-y_{L}\right) \psi_{L}}$. Therefore the profit function has a global maximum in this point. The corresponding value of $w_{L}^{\prime}$ follows from evaluating equation (24):

$$
w_{L}^{\prime}=\frac{-\psi_{L} y_{L}}{1-\psi_{L}}\left(\frac{\psi_{L} \log \kappa}{1-\kappa}+1\right)
$$


One can check that $\lim _{\psi_{L} \rightarrow 0} w_{L}^{\prime}=0, \lim _{\psi_{L} \rightarrow 0} \frac{\partial w_{L}^{\prime}}{\partial \psi_{L}}=-y_{L}<0$ and, by applying l'Hospital's Rule twice, $\lim _{\psi_{L} \rightarrow 1} w_{L}^{\prime}=\frac{1-y_{L}}{2}>0$ (see Figure 16). Therefore, it depends on the equilibrium value $\psi_{L}^{*}$ whether a profitable deviation exists. For $\psi_{L}^{*}$ close to 0 the optimal value for $w_{L}^{\prime}$ is negative. Given the fact that

$\frac{\partial \pi_{L}^{\prime}}{\partial \phi_{L}^{\prime}}<0$ for $\phi_{L}^{\prime}>-\log \kappa$ and that $\frac{\partial w_{L}^{\prime}}{\partial \phi_{L}^{\prime}}>0 \forall \phi_{L}^{\prime}>0$, this implies that low type firms have no incentive to post a wage that is different from 0 . On the other hand, for $\psi_{L}^{*}$ close to 1 , it is profitable for a low firm to deviate by posting a wage that is strictly positive. It straightforward to show that both cases can occur. For example, $\psi_{L}^{*} \rightarrow 0$ if $\theta_{H} \rightarrow 0, \theta_{L} \rightarrow 0$ and $y_{L} \rightarrow 1$, while $\psi_{L}^{*} \rightarrow 1$ if $\theta_{H} \rightarrow \hat{\theta}_{H}$ where $\hat{\theta}_{H}$ is such that $\frac{\hat{\theta}_{H}^{2}}{4}\left(1-\exp \left(-\frac{2}{\hat{\theta}_{H}}\right)\right)^{2}=y_{L}$.

\section{A.3 Proof of Proposition 5}

Proof. The function $1-e^{-\phi_{H}}$ is strictly positive and strictly increasing $\forall \phi_{H}>0$. The same is true for the function $1-\psi_{H}=1-\frac{1}{\phi_{H}}\left(1-e^{-\phi_{H}}\right)$. Therefore, the revenue for the high firm $\left(1-e^{-\phi_{H}}\right)\left(1-\psi_{H}\right)$ is a strictly increasing function of $\phi_{H}$ with

$$
\lim _{\phi_{H} \rightarrow 0}\left(1-e^{-\phi_{H}}\right)\left(1-\psi_{H}\right)=0
$$

and

$$
\lim _{\phi_{H} \rightarrow \infty}\left(1-e^{-\phi_{H}}\right)\left(1-\psi_{H}\right)=1 .
$$

This implies that the condition (16) uniquely identifies a value $\phi_{H}^{*}>0$ for any $0<c_{H}<1$.

Since $\left(1-e^{-\phi_{L}}\right)\left(1-\psi_{L}\right)<1$, a necessary condition for condition (17) to hold is that $p_{L}>c_{L}$. Assume for the moment that $p_{L}$ is exogenously given such that this condition is satisfied. In that case any value $0<c_{L}<1$ uniquely identifies a value $\phi_{L}^{*}$ as a function of $p_{L}$, i.e. $\phi_{L}^{*}\left(p_{L}\right)$. Since $\left(1-e^{-\phi_{L}}\right)\left(1-\psi_{L}\right)$ is strictly increasing in $\phi_{L}, \phi_{L}^{*}\left(p_{L}\right)$ is strictly decreasing in $p_{L}$ with $\lim _{p_{L} \rightarrow c_{L}} \phi_{L}^{*}\left(p_{L}\right)=\infty$ and $\lim _{p_{L} \rightarrow \infty} \phi_{L}^{*}\left(p_{L}\right)=0$. Using this, it follows directly that $\psi_{L}\left(\phi_{L}^{*}\left(p_{L}\right)\right)$ and $\psi_{L}\left(\phi_{L}^{*}\left(p_{L}\right)\right)^{2} p_{L}$ are both strictly increasing in $p_{L}$ and that

$$
\lim _{p_{L} \rightarrow c_{L}} \psi_{L}\left(\phi_{L}^{*}\left(p_{L}\right)\right)^{2} p_{L}=0
$$

and

$$
\lim _{p_{L} \rightarrow \infty} \psi_{L}\left(\phi_{L}^{*}\left(p_{L}\right)\right)^{2} p_{L}=\infty .
$$

This implies that given $\phi_{H}^{*}$ and $\phi_{L}^{*}\left(p_{L}\right)$ there exists a unique value $p_{L}^{*}>c_{L}$ such that the indifference condition is satisfied.

Let $\phi_{L}^{*}=\phi_{L}^{*}\left(p_{L}^{*}\right), \psi_{H}^{*}=\psi_{H}\left(\phi_{H}^{*}\right)$ and $\psi_{L}^{*}=\psi_{L}\left(\phi_{L}^{*}\left(p_{L}^{*}\right)\right)$. Then

$$
\lim _{q_{H H} \rightarrow 0} \frac{1-\lambda}{\lambda} \frac{q_{H H}}{1-q_{H H}} \frac{1-\left(1-\psi_{H}^{*}\right)^{2}}{1-\left(1-\psi_{L}^{*}\right)^{2}}=0,
$$

while

$$
\lim _{q_{H H} \rightarrow 1} \frac{1-\lambda}{\lambda} \frac{q_{H H}}{1-q_{H H}} \frac{1-\left(1-\psi_{H}^{*}\right)^{2}}{1-\left(1-\psi_{L}^{*}\right)^{2}}=\infty
$$


and

$$
\frac{d}{d q_{H} H} \frac{q_{H H}}{1-q_{H H}}=\frac{1}{\left(1-q_{H H}\right)^{2}}>0 .
$$

The Intermediate Value Theorem implies that there exists a unique value $0<q_{H H}^{*}<1$ such that $p_{L}^{*}$ equals the MRS $\frac{1-\lambda}{\lambda} \frac{q_{H H}^{*}}{1-q_{H}^{*}} \frac{1-\left(1-\psi_{H}^{*}\right)^{2}}{1-\left(1-\psi_{L}^{*}\right)^{2}}$. Using $q_{H H}^{*}, \phi_{H}^{*}$ and $\phi_{L}^{*}$, it is straightforward to determine $\theta_{H}^{*}$ and $\theta_{L}^{*}$. Now, the equilibrium is defined by $p_{L}^{*}, \theta_{H}^{*}, \theta_{L}^{*}$, and $q_{H H}^{*}$.

\section{A.4 Proof of Proposition 6}

Proof. Let $p_{L}^{*}$ and $\hat{p}_{L}^{*}$ be equilibrium prices with $p_{L}^{*}>\hat{p}_{L}^{*}$. Using the indifference condition this implies $\psi_{L}^{*}<\hat{\psi}_{L}^{*}$, which by the definition of $\psi_{i}$ is equivalent to $\phi_{L}^{*}>\hat{\phi}_{L}^{*}$. Using the inequalities $p_{L}^{*}>\hat{p}_{L}^{*}$ and $\phi_{L}^{*}>\hat{\phi}_{L}^{*}$, and the fact that $\left(1-e^{-\phi_{L}}\right)\left(1-\frac{1}{\phi_{L}}\left(1-e^{-\phi_{L}}\right)\right)$ is strictly increasing in $\phi_{L}$, one can derive that

$$
\begin{aligned}
\hat{c}_{L} & =\left(1-e^{-\hat{\phi}_{L}^{*}}\right)\left(1-\frac{1}{\hat{\phi}_{L}^{*}}\left(1-e^{-\hat{\phi}_{L}^{*}}\right)\right) \hat{p}_{L}^{*} \\
& <\left(1-e^{-\phi_{L}^{*}}\right)\left(1-\frac{1}{\phi_{L}^{*}}\left(1-e^{-\phi_{L}^{*}}\right)\right) p_{L}^{*}=c_{L}
\end{aligned}
$$

Likewise, one can show that $p_{L}^{*}<\hat{p}_{L}^{*}$ implies $\psi_{L}^{*}>\hat{\psi}_{L}^{*}, \phi_{L}^{*}<\hat{\phi}_{H}^{*}$, and $\hat{c}_{L}>c_{L}$ and that $p_{L}^{*}=\hat{p}_{L}^{*}$ implies $\psi_{L}^{*}=\hat{\psi}_{L}^{*}, \phi_{L}^{*}=\hat{\phi}_{H}^{*}$, and $\hat{c}_{L}=c_{L}$. Since we have listed all possibilities, we can invert this result and state that $c_{L}>\hat{c}_{L}$ implies $\phi_{L}^{*}>\hat{\phi}_{L}^{*}, \psi_{L}^{*}<\hat{\psi}_{L}^{*}$, and $p_{L}^{*}>\hat{p}_{L}^{*}$. Hence, $\phi_{L}^{*}$ and $p_{L}^{*}$ are strictly increasing in $c_{L}$. The equilibrium value of $\phi_{H}^{*}$ is determined by the condition (16) only and therefore not affected by a change in $c_{L}$

Substituting the indifference condition in the fourth condition and solving for $q_{H H}^{*}$ yields the following expression

$$
q_{H H}^{*}=\frac{2 \lambda \psi_{H}^{*}}{2 \lambda\left(\psi_{H}^{*}-\psi_{L}^{*}\right)+\left(2-\psi_{H}^{*}\right) \psi_{L}^{*}} .
$$

From this we can derive

$$
\begin{aligned}
\frac{d q_{H H}^{*}}{d c_{L}} & =\frac{\partial q_{H H}^{*}}{\partial \psi_{L}^{*}} \frac{\partial \psi_{L}^{*}}{\partial c_{L}}+\frac{\partial q_{H H}^{*}}{\partial \psi_{H}^{*}} \frac{d \psi_{H}^{*}}{d c_{L}} \\
& =\frac{\partial q_{H H}^{*}}{\partial \psi_{L}^{*}} \frac{\partial \psi_{L}^{*}}{\partial c_{L}}>0 .
\end{aligned}
$$

Now we have

$$
\frac{\partial \phi_{H}^{*}}{\partial c_{L}}=\frac{\partial \phi_{H}^{*}}{\partial q_{H H}^{*}} \frac{\partial q_{H H}^{*}}{\partial c_{L}}+\frac{\partial \phi_{H}^{*}}{\partial \theta_{H}^{*}} \frac{\partial \theta_{H}^{*}}{\partial c_{L}} .
$$

As shown above, $\frac{\partial \phi_{H}^{*}}{\partial c_{L}}=0$, while $\frac{\partial \phi_{H}^{*}}{\partial q_{H}^{*}}>0, \frac{\partial q_{H H}^{*}}{\partial c_{L}}>0$ and $\frac{\partial \phi_{H}^{*}}{\partial \theta_{H}^{*}}<0$. This implies $\frac{\partial \theta_{H}^{*}}{\partial c_{L}}>0$.

Likewise, we have

$$
\frac{\partial \phi_{L}^{*}}{\partial c_{L}}=\frac{\partial \phi_{L}^{*}}{\partial q_{H H}^{*}} \frac{\partial q_{H H}^{*}}{\partial c_{L}}+\frac{\partial \phi_{L}^{*}}{\partial \theta_{L}^{*}} \frac{\partial \theta_{L}^{*}}{\partial c_{L}}
$$

where $\frac{\partial \phi_{L}^{*}}{\partial c_{L}}>0, \frac{\partial \phi_{L}^{*}}{\partial q_{H}^{*}}<0, \frac{\partial q_{H H}^{*}}{\partial c_{L}}>0$ and $\frac{\partial \phi_{L}^{*}}{\partial \theta_{L}^{*}}<0$. This implies $\frac{\partial \theta_{L}^{*}}{\partial c_{L}}<0$. 


\section{B Figures}

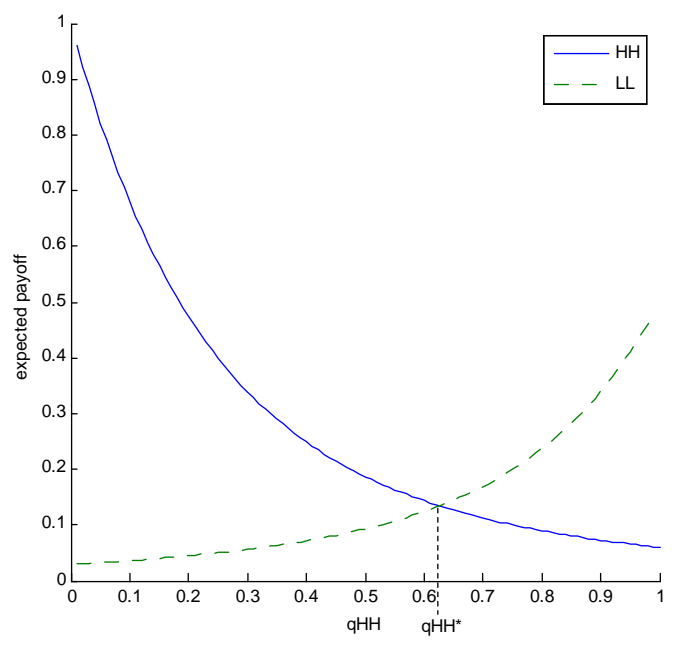

Figure 1: Expected payoff of playing $H H$ and $L L$ for $\theta_{H}=\theta_{L}=1$ and $y_{L}=0.5$ 


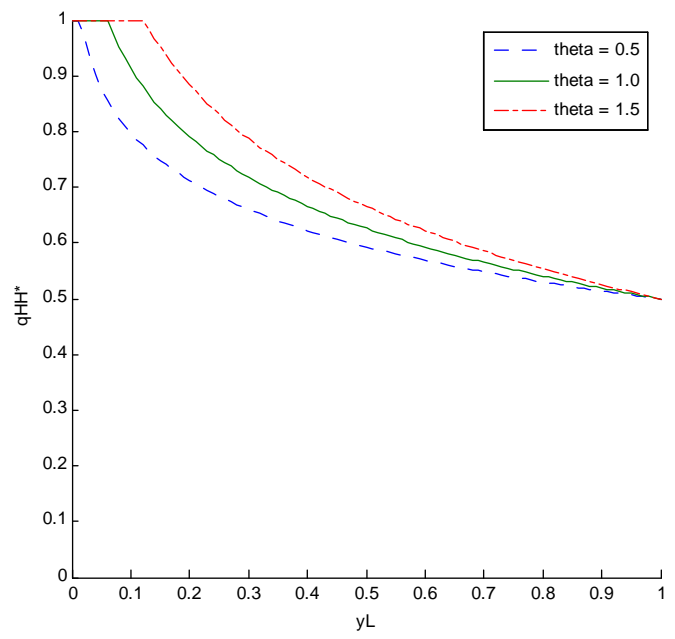

Figure 2: $q_{H H}^{*}$ as a function of $y_{L}$ for several values of $\theta_{H}=\theta_{L}=\frac{1}{2} \theta$.

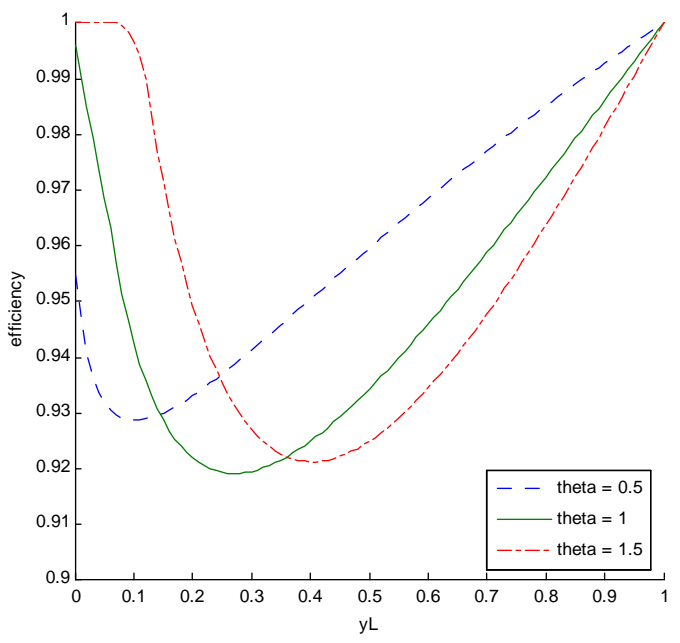

Figure 3: Efficiency of the decentralized equilibrium $\left(Y^{*} / Y^{* *}\right)$ as a function of $y_{L}$ for several values of $\theta_{H}=\theta_{L}=\frac{1}{2} \theta$. 


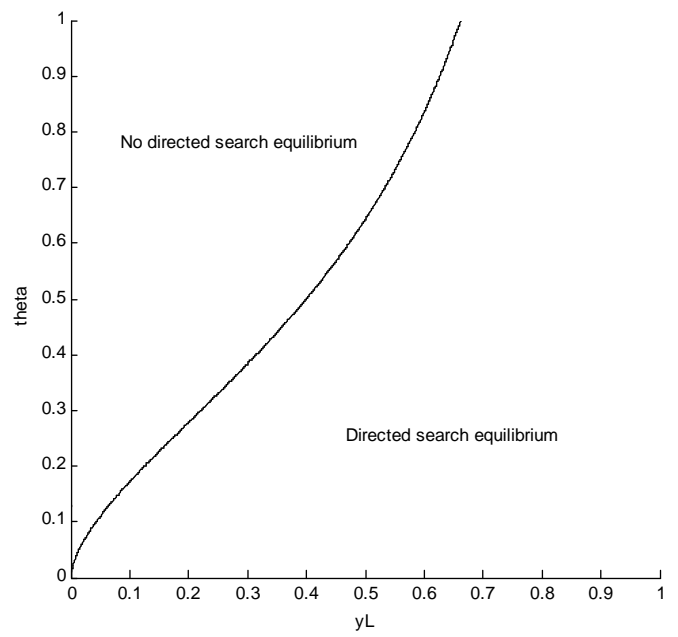

Figure 4: Combinations of $y_{L}$ and $\theta_{H}=\theta_{L}=\frac{1}{2} \theta$ for which the equilibrium outcomes of a random search model and a directed search model coincide.

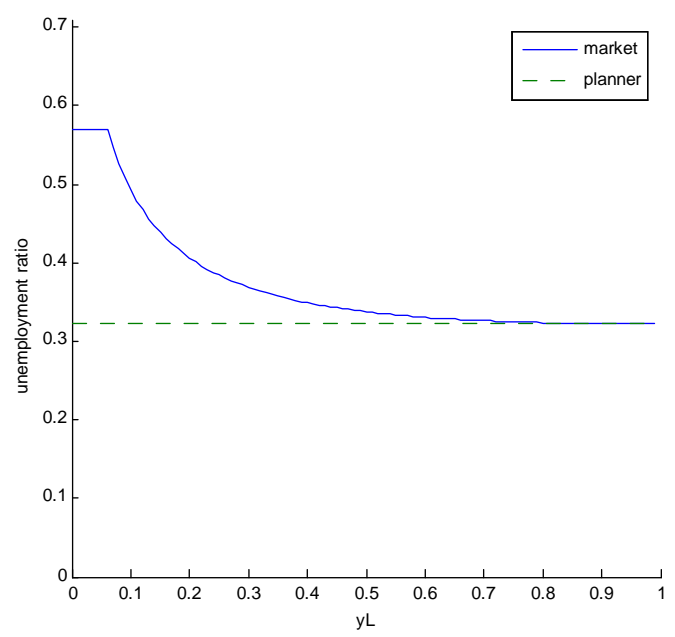

Figure 5: Unemployment ratio as a function of $y_{L}$ for $\theta_{H}=\theta_{L}=0.5$. 


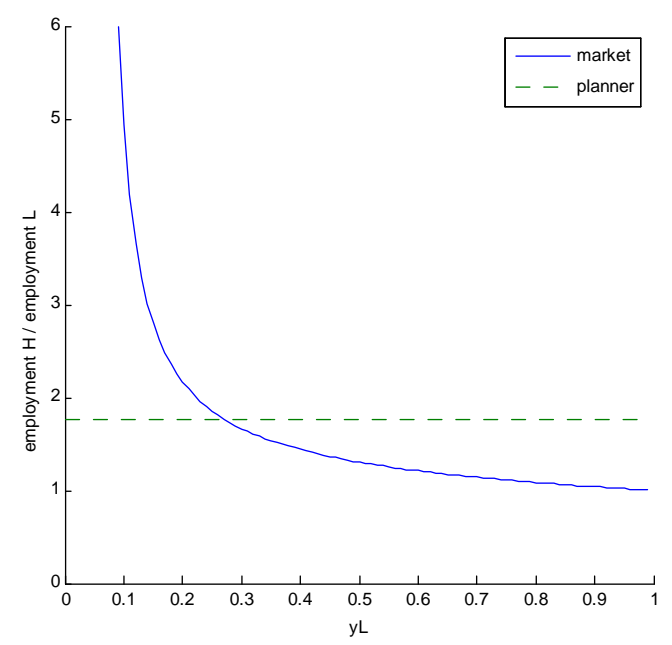

Figure 6: Ratio between employment in the high and low sector as a function of $y_{L}$ for $\theta_{H}=\theta_{L}=\frac{1}{2}$.

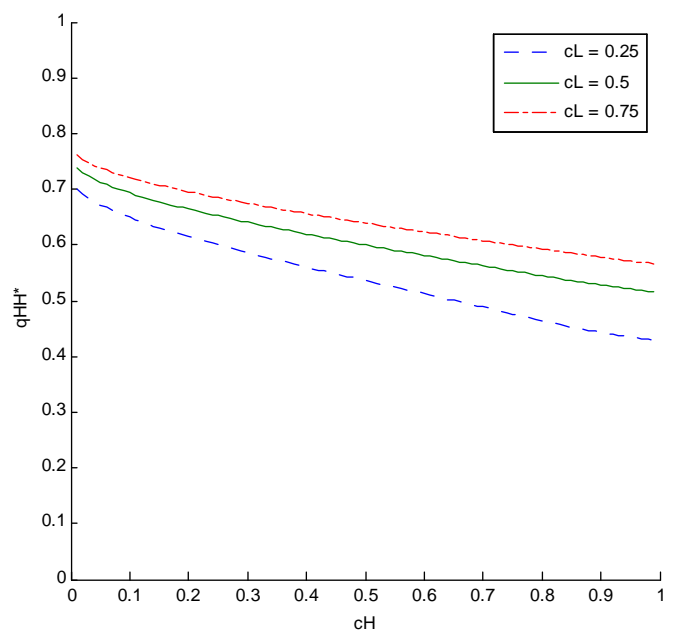

Figure 7: $q_{H H}^{*}$ as a function of $c_{H}$ for $\lambda=0.6$ and several values of $c_{L}$. 


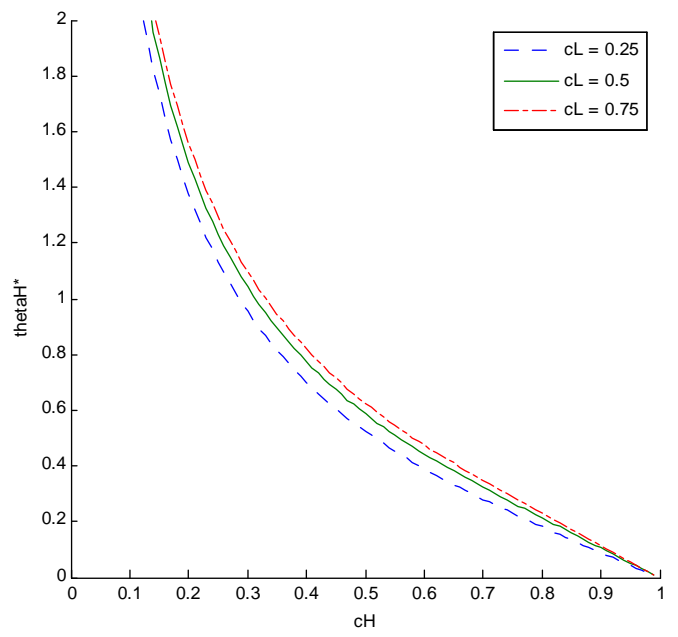

Figure 8: $\theta_{H}^{*}$ as a function of $c_{H}$ for $\lambda=0.6$ and several values of $c_{L}$.

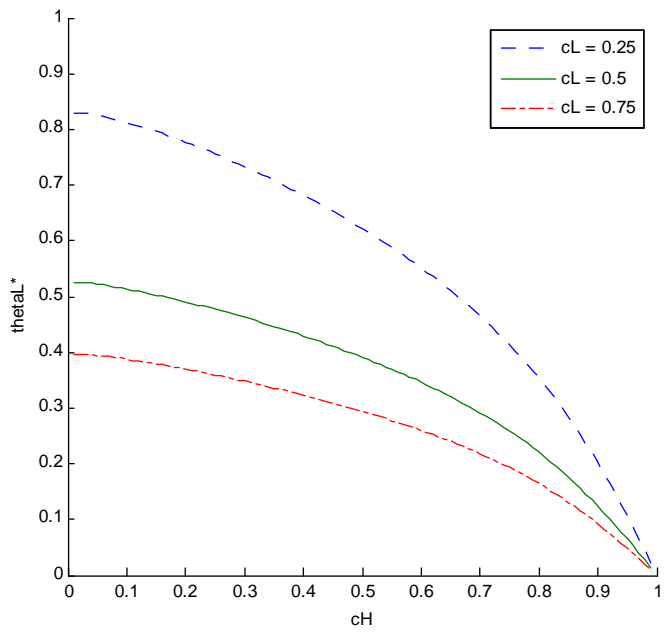

Figure 9: Equilibrium value $\theta_{L}^{*}$ as a function of $c_{H}$ for $\lambda=0.6$ and several values of $c_{L}$. 


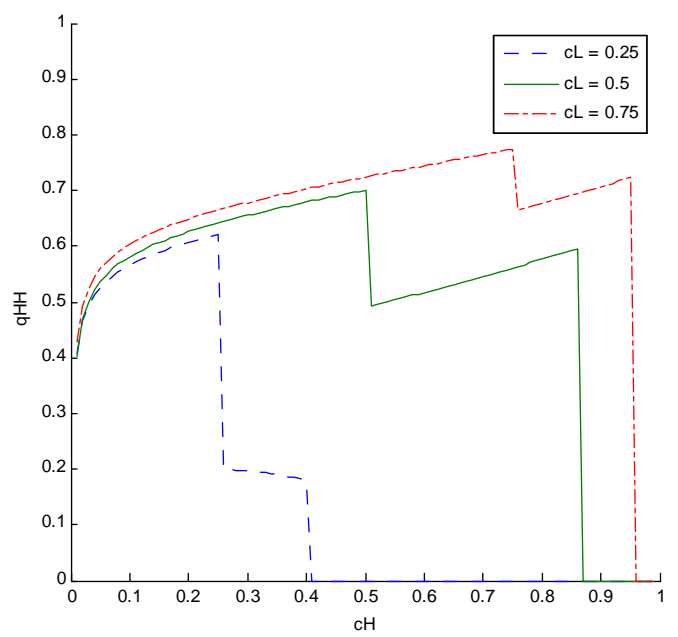

Figure 10: $q_{H H}^{* *}$ as a function of $c_{H}$ for $\lambda=0.6$ and several values of $c_{L}$.

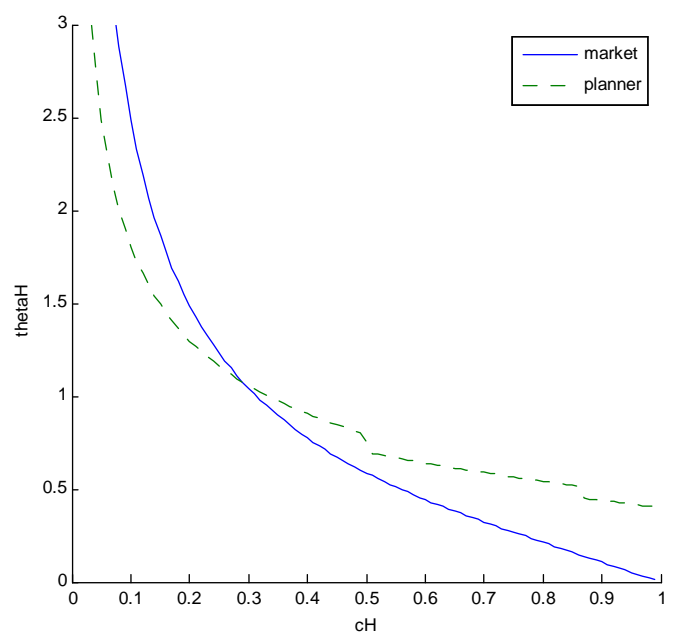

Figure 11: The number of high firms in the market as a function of $c_{H}$ for $\lambda=0.6$ and $c_{L}=0.5$. 


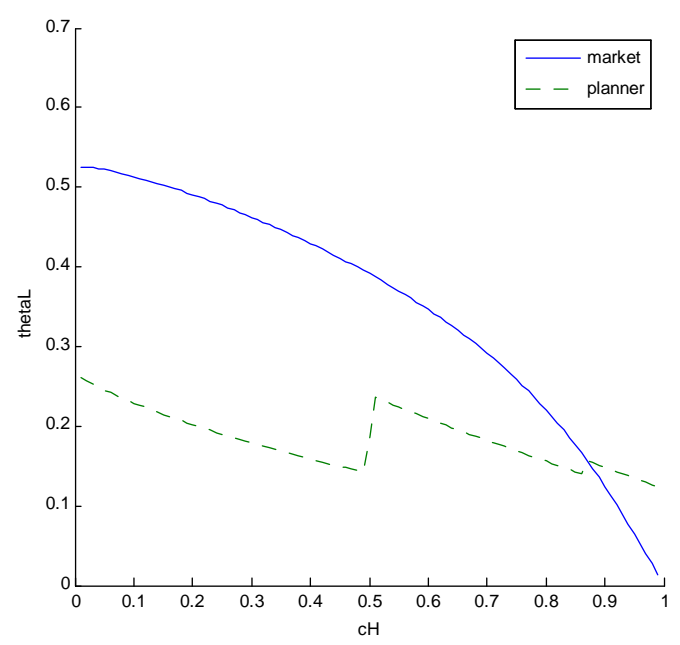

Figure 12: The number of low firms in the market as a function of $c_{H}$ for $\lambda=0.6$ and $c_{L}=0.5$.

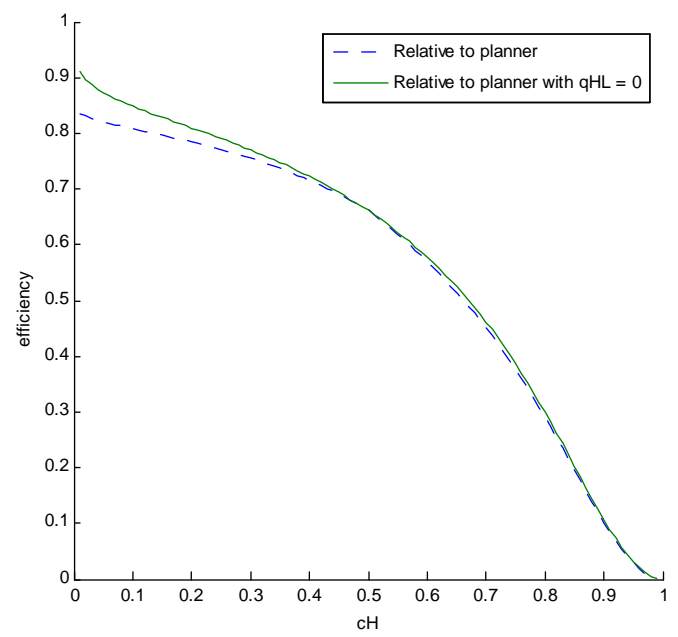

Figure 13: Efficiency of the decentralized equilibrium as a function of $c_{H}$ for $\lambda=0.6$ and $c_{L}=0.5$. 


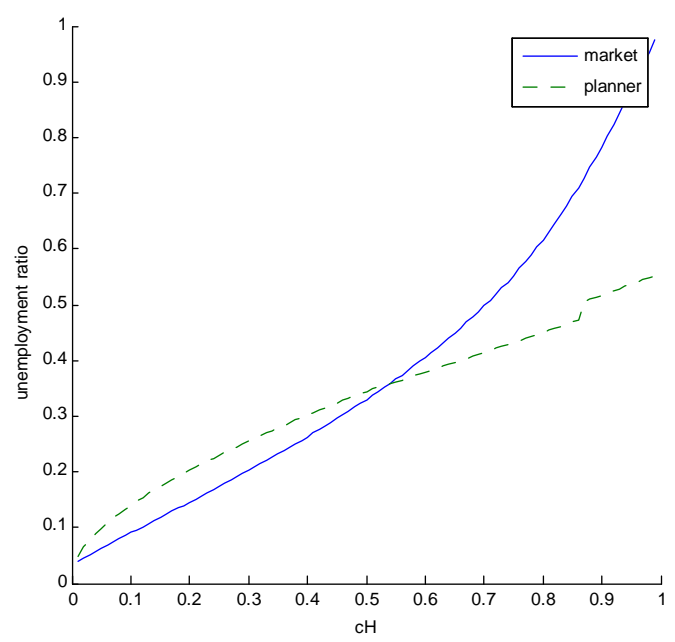

Figure 14: Unemployment ratio for $c_{L}=0.5$ and $\lambda=0.6$ and $c_{L}=0.5$.

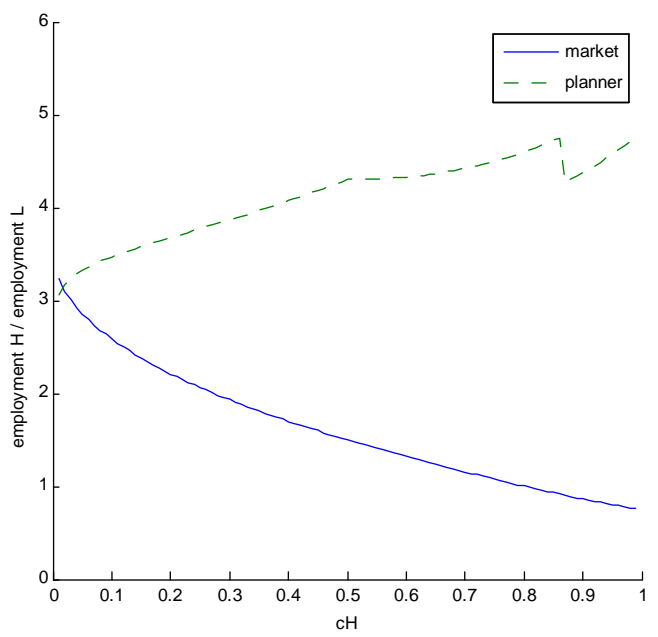

Figure 15: Ratio of employment in high and low sector as a function of $c_{H}$ for $\lambda=0.6$ and $c_{L}=0.5$. 


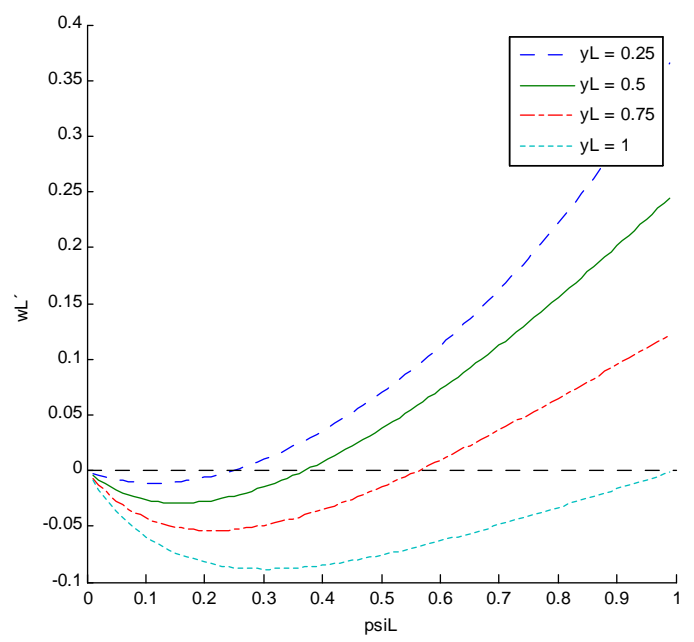

Figure 16: $w_{L}^{\prime}$ as a function of $\psi_{L}$ for several values of $y_{L}$. Positive values of $w_{L}^{\prime}$ imply that a profitable deviation exists for a low type firm. 